\title{
Synthesis and Photophysical Properties of Benzotriazole-Derived Unnatural $\alpha$-Amino Acids
}

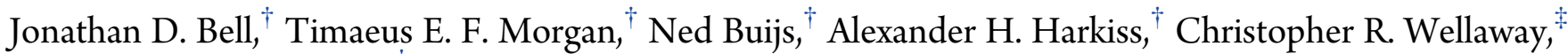 \\ and Andrew Sutherland ${ }^{* \dagger}+0$
}

${ }^{\dagger}$ WestCHEM, School of Chemistry, The Joseph Black Building, University of Glasgow, Glasgow G12 8QQ U.K.

${ }^{\ddagger}$ Medicinal Chemistry, GlaxoSmithKline Medicines Research Centre, Gunnels Wood Road, Stevenage SG1 2NY, U.K.

\section{Supporting Information}

ABSTRACT: The synthesis of a new class of benzotriazole-derived $\alpha$ amino acid is described using a highly efficient nucleophilic aromatic substitution of ortho-fluoronitrobenzenes with L-3-aminoalanine and a polymer-supported nitrite reagent-mediated diazotization and cyclization of the subsequent 1,2-aryldiamines as the key steps. Further functionalization of the benzotriazole unit by preparation of halogenated analogues and Suzuki-Miyaura cross-coupling with aryl boronic acids allowed the synthesis of $\alpha$-amino acids with conjugated side chains.

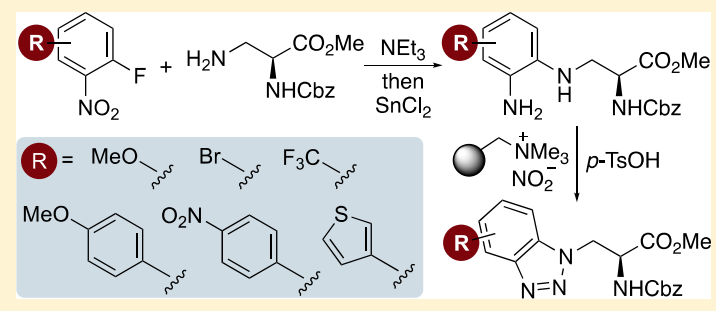
Analysis of the photophysical properties of these $\alpha$-amino acids revealed that incorporation of electron-rich substituents results in charge-transfer-based, fluorescent compounds with MegaStokes shifts.

\section{INTRODUCTION}

For many years, nonproteinogenic $\alpha$-amino acids have been deemed as highly important targets for a range of applications. ${ }^{1}$ In synthetic chemistry, they are widely used as chiral precursors, ligands, and catalysts and are found to be components of many natural products and pharmaceutically relevant compounds. ${ }^{2}$ In medicinal chemistry and the life sciences, unnatural $\alpha$-amino acids are commonly used as enzyme inhibitors or as probes to study biological mechanisms and protein structure and function. ${ }^{3}$ In this regard, there have been significant efforts on the design and development of fluorescent unnatural $\alpha$-amino acids as tools for a range of chemical biology applications. ${ }^{4}$ Many important fluorescent $\alpha$ amino acid probes have been rapidly prepared by incorporating a chromophore within the side chain of L-3-aminoalanine. ${ }^{5}$ These include the environment-sensitive and charge-transferbased dimethylaminophthalimide $\mathbf{1}^{6}$ and the related naphthalimide analogue 2, which have been used for sensing protein-protein interactions (Figure 1). Other examples include 6-acetylnaphthalene-substituted $\alpha$-amino acid 3, which has been genetically incorporated into proteins and used to study protein-ligand and protein-protein interactions, ${ }^{8}$ while dansyl-labeled L-3-aminoalanine derivative 4 has also been widely used to study biomolecular interactions and protein structural dynamics. ${ }^{9}$ Various benzo-fused heterocycles have also been attached to the side chain of L-3aminoalanine and used as biological probes. For example, a nitro-substituted benzoxadiazole analogue 5 was found to be an environment-sensitive fluorophore and used to study the structure and function of a neurokinin-2 receptor. ${ }^{10}$ An azide analogue of L-3-aminoalanine was used in a click-type reaction to attach a benzothiadiazole unit, resulting in a highly<smiles>CC(=O)c1ccc2cc(NCC(N)C(=O)O)ccc2c1</smiles><smiles>CN(C)c1cccc2c(S(=O)(=O)NCC(N)C(=O)O)cccc12</smiles><smiles>NC(CNc1ccc([N+](=O)[O-])c2nonc12)C(=O)O</smiles>

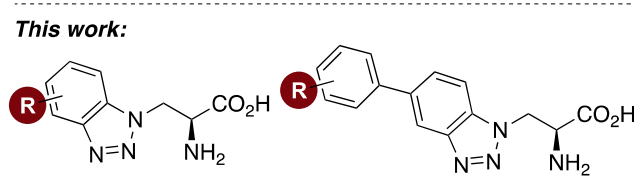

Figure 1. Unnatural $\alpha$-amino acids derived from L-3-aminoalanine.

fluorescent $\alpha$-amino acid 6 that is emitted in the visible region with a good quantum yield. ${ }^{11}$

Previously, we have described the development of various classes of fluorescent $\alpha$-amino acids incorporating a wide range of chromophores, including heterocycle-based systems.

Received: June 24, 2019

Published: July 25, 2019 
While these studies have identified highly fluorescent, environment-sensitive probes that can be incorporated into peptides using solid phase peptide synthesis, we were interested in developing novel benzo-fused heterocyclic-based targets that could be accessed in relatively few steps from a common L-3-aminoalanine derivative and diversified at a late stage to access chromophores with a range of photophysical properties. Here, we report a general synthetic approach to benzotriazole-derived $\alpha$-amino acids (Figure 1) from L-3aminoalanine using a nucleophilic aromatic substitution reaction with ortho-fluoronitrobenzenes and a mild one-pot diazotization and cyclization process of subsequent 1,2aryldiamines to form the key triazole ring. Tuning the photophysical properties of these novel chromophores through the incorporation of additional arene units using the SuzukiMiyaura reaction with various halogenated benzotriazoles is also described.

\section{RESULTS AND DISCUSSION}

Our approach to $\alpha$-amino acids bearing benzotriazole side chains is shown in Scheme 1. Initially, it was proposed that a

Scheme 1. Proposed Synthesis of Benzotriazole-Derived $\alpha$ Amino Acids
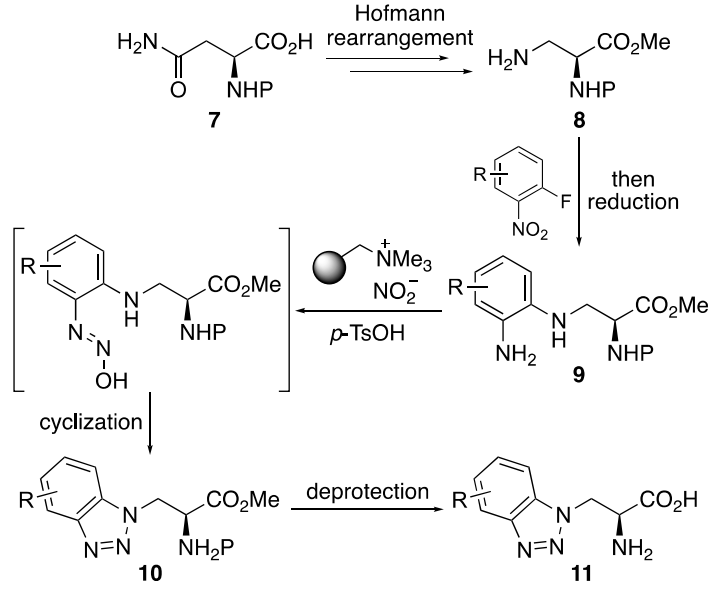

suitably protected L-3-aminoalanine derivative 8 could be quickly accessed from L-asparagine via a Hofmann rearrangement. Nucleophilic aromatic substitution of ortho-2-fluoronitrobenzenes with $\mathbf{8}$, followed by nitro group reduction, would give a range of 1,2-aryldiamines 9 incorporating an $\alpha$-amino acid component. The next stage required activation and cyclization of the 1,2-aryldiamines via a diazo intermediate. Filimonov and co-workers have previously shown that anilines can be activated as diazonium tosylate salts under mild conditions, using a polymer-supported nitrite reagent and $p$ tosic acid. ${ }^{13,14}$ In addition to the mild conditions, the advantages of this approach include an operationally simple procedure and straightforward work-up and purification of the products. Various reports have demonstrated the synthetic utility of this activation process for one-pot multistep reaction of arenes. ${ }^{13-15}$ In this project, the aim was to demonstrate that substrates such as 9 could be activated and cyclized for the general synthesis of benzotriazole-derived $\alpha$-amino acids $\mathbf{1 0}$. Deprotection would then allow access to parent $\alpha$-amino acids 11.

The first stage of the project investigated a short, scalable synthesis of an L-3-aminoalanine derivative. This was achieved in two steps and quantitative yield from commercially available $\mathrm{N}$-Cbz-L-asparagine (12) via a Hofmann rearrangement using (diacetoxyiodo)benzene, ${ }^{16}$ followed by esterification with thionyl chloride and methanol (Scheme 2). ${ }^{17}$ The resulting

Scheme 2. Synthesis of Benzotriazole-Derived $\alpha$-Amino Acids 18a-f $\mathbf{f}^{a}$

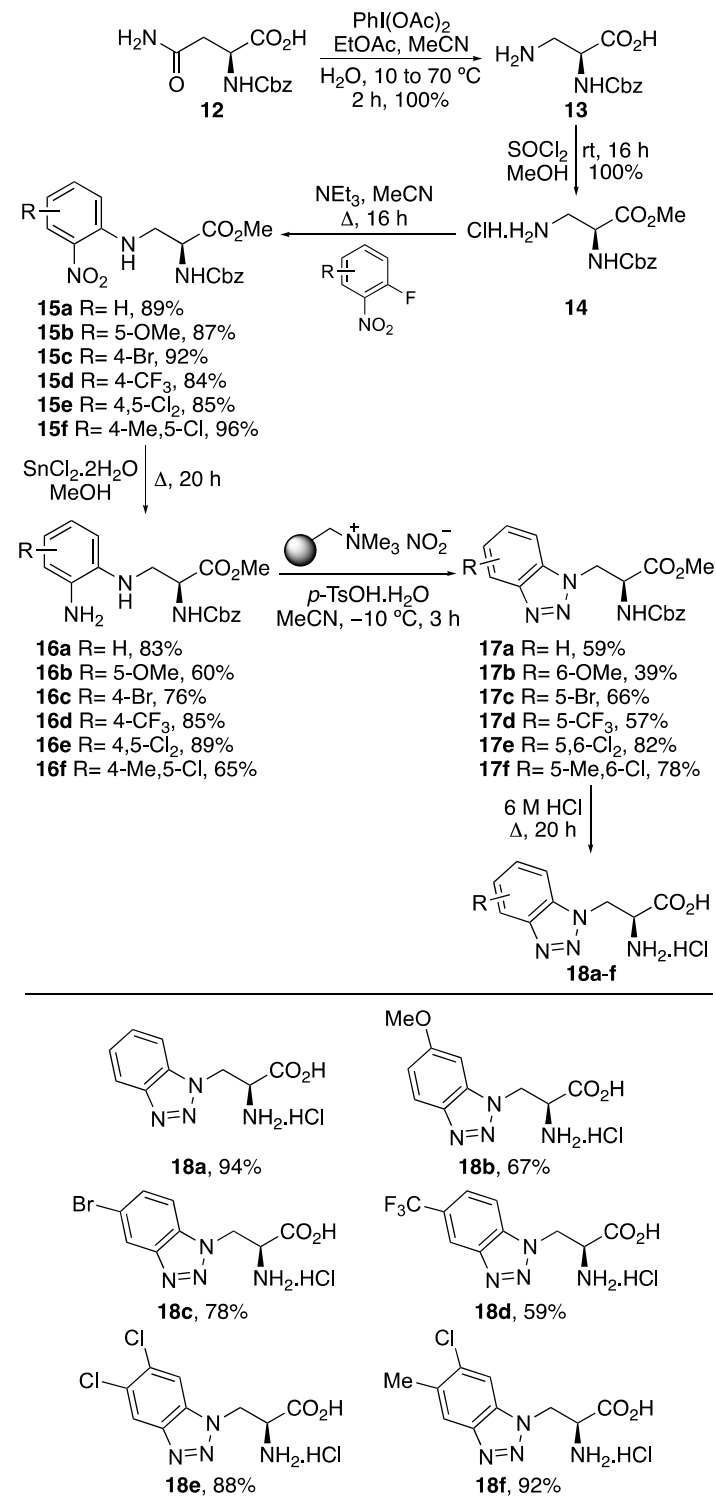

${ }^{a}$ Isolated yields are shown.

L-3-aminoalanine derivative $\mathbf{1 4}$ was used in a nucleophilic aromatic substitution reaction with a series of orthofluoronitrobenzenes in the presence of triethylamine. ${ }^{18}$ This reaction gave consistently high yields with both electron-rich and electron-deficient ortho-fluoronitrobenzenes. Reduction of the nitro moiety of compounds $15 \mathbf{a}-\mathbf{f}$, under chemoselective conditions with tin dichloride, proceeded smoothly to give $\alpha$ amino acid-substituted 1,2-aryldiamines 16a-f. Activation and subsequent cyclization of these key intermediates to the corresponding benzotriazoles using the polymer-supported nitrite reagent and $p$-tosic acid were then investigated. ${ }^{15} \mathrm{~A}$ brief optimization study showed that performing this transformation at $-10{ }^{\circ} \mathrm{C}$ generated the majority of benzotriazoles $17 \mathbf{a}$ and $17 \mathrm{c}-\mathrm{f}$ cleanly and, in good to high yields $(57-82 \%)$. Synthesis 
of benzotriazole $\mathbf{1 7 b}$ from the more reactive, electron-rich $p$ methoxyphenyl substrate $\mathbf{1 6 b}$ was found to be less efficient (39\%). ${ }^{19}$ Deprotection of $\mathbf{1 7} \mathbf{a}-\mathbf{f}$ was then conducted in a onepot process under acidic conditions. Purification by recrystallization gave $\alpha$-amino acids $\mathbf{1 8 a}-\mathbf{f}$ as the hydrochloride salts in 59-94\% yields.

Having shown that $\alpha$-amino acid-substituted 1,2-aryldiamines could be converted to the corresponding benzotriazoles under the mild conditions of the diazotization and cyclization process, the next stage of this project was to demonstrate that these heterocyclic motifs could be further functionalized, allowing the late-stage preparation of a more diverse series of novel $\alpha$-amino acids. With the aim of producing fluorescent $\alpha$ amino acids, we sought to extend the conjugation of the benzotriazole heterocycle. This was achieved using a SuzukiMiyaura reaction with bromide analogue $17 \mathrm{c}$ and a range of aryl boronic acids (Scheme 3). ${ }^{20}$ To fully understand how

Scheme 3. Synthesis of 5-Aryl Benzotriazole-Derived $\alpha$ Amino Acids 21a-f $\mathbf{f}^{a}$
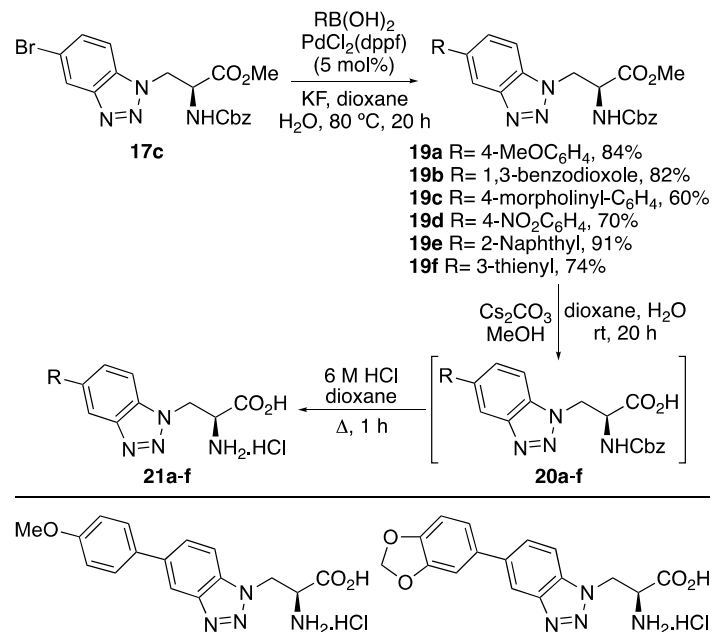

21a, $77 \%$ 21b, $89 \%$
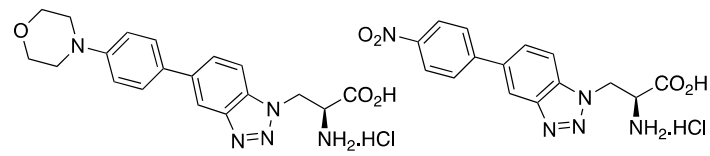
21c, $97 \%$

21d, $93 \%$
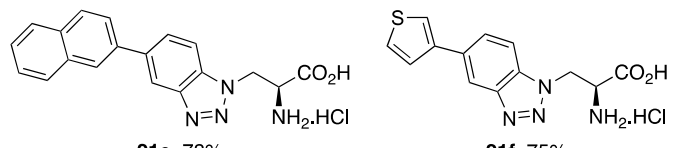

21e, $78 \%$

${ }^{a}$ Isolated yields are shown.

substituents might affect the photophysical properties of the $\alpha$ amino acids, aryl boronic acids with electron-deficient or electron-rich substituents were investigated. Bromide analogue $17 \mathrm{c}$ was found to be an excellent coupling partner, allowing the preparation of a small library of conjugated benzotriazolebased $\alpha$-amino acids in good to excellent yields (60-91\%). Initial attempts at deprotecting amino acids $19 a-f$ under acidic conditions previously utilized (Scheme 2; reflux, $20 \mathrm{~h}$ ) did produce the target compounds, but less efficiently than expected. Therefore, a milder, two-step approach was utilized instead, involving base hydrolysis of the ester moiety, followed by a rapid acid-mediated deprotection of the amino group.

This gave the final $\alpha$-amino acids 21 a-f in $75-97 \%$ yields over the two steps.

A final target was prepared by investigating the functionalization of the methoxy-substituted benzotriazole $\mathbf{1 7 b}$ (Scheme 4). We have previously reported the highly regioselective

Scheme 4. Four-Step Synthesis of Benzotriazole-Derived $\alpha$ Amino Acid $24^{a}$
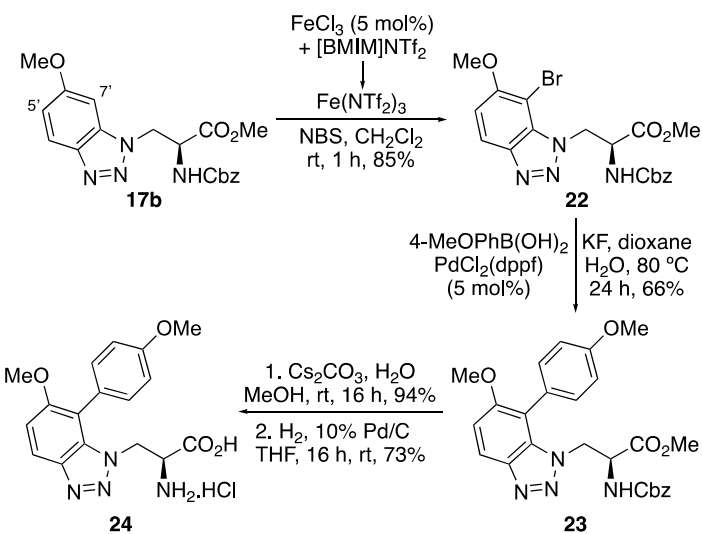

${ }^{a}$ Isolated yields are shown.

halogenation of activated arenes using various $N$-halosuccinimides and the super Lewis acid, iron triflimide, easily prepared from iron(III) chloride and the commercially available ionic liquid, [BMIM] $\mathrm{NTf}_{2} .{ }^{21}$ With the most activated para-position blocked, ${ }^{22}$ it was expected that of the two ortho-positions, iron triflimide-catalyzed bromination of $\mathbf{1 7} \mathbf{b}$ with $\mathrm{N}$-bromosuccinimide (NBS) would occur at the least hindered C-5' position. Surprisingly, bromination occurred at the more hindered C- $7^{\prime}$ position, giving 22 after $1 \mathrm{~h}$ as the sole product in $85 \%$ yield. This result was confirmed by ${ }^{1} \mathrm{H}$ NMR spectroscopy of $\mathbf{2 2}$ which clearly showed two separate aromatic hydrogen atoms with an ortho-coupling constant $(9.0 \mathrm{~Hz})$. We propose that the amino acid moiety of $\mathbf{1 7} \mathbf{b}$ may be involved in directing the iron(III)-activated NBS complex to the $\mathrm{C}-7^{\prime}$ position. The brominated benzotriazole unit was then used to extend the conjugation of the heterocyclic unit. With the aim of preparing an electron-rich chromophore with charge-transfer properties, 22 was subjected to a Suzuki-Miyaura reaction with 4methoxyphenylboronic acid. This gave coupled product 23 in $66 \%$ yield. Deprotection of 23 was then conducted in a twostage process. As before, the methyl ester was hydrolyzed in high yield using cesium carbonate. Hydrogenation using $10 \%$ $\mathrm{Pd} / \mathrm{C}$ proved to be the most efficient method for removal of the Cbz-protecting group. Formation of the hydrochloride salt and recrystallization gave $\alpha$-amino acid 24 in 73\% yield.

Following the synthesis of the benzotriazole-derived $\alpha$ amino acids, the photophysical properties were measured for each compound. The UV/visible absorption and photoluminescence spectra of the $\alpha$-amino acids were recorded in methanol at a concentration of $1 \times 10^{-5} \mathrm{M}$. As expected, the nonconjugated benzotriazoles $18 \mathbf{a}-\mathbf{f}$ and the electron-deficient 5-( $p$-nitrophenyl)benzotriazole 21d displayed no fluorescence in methanol. In contrast, the electron-rich, aryl-conjugated benzotriazoles 21a-c, 21e, 21f, and 24 all showed strong fluorescence with emission maxima in the visible region, ranging from 384 to $454 \mathrm{~nm}$ (Figure $2 \mathrm{~b}$ and Table 1 ). ${ }^{23}$ These compounds also possess MegaStokes shifts that are important in avoiding reabsorption. The nonrigid nature of these 

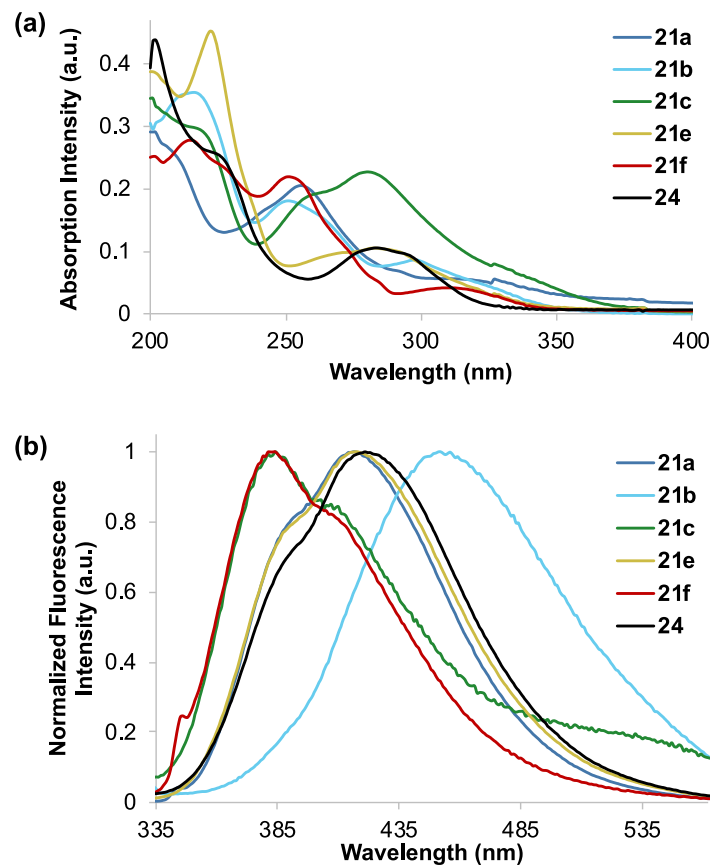

Figure 2. (a) Absorption spectra of 21a-c, 21e, 21f, and 24, recorded at $1 \times 10^{-5} \mathrm{M}$ in methanol. (b) Emission spectra of 21a-c, 21e, 21f, and 24 , recorded at $1 \times 10^{-5} \mathrm{M}$ in methanol.

Table 1. Photophysical Data of Benzotriazole-Derived $\alpha$ Amino Acids 21a-c, 21e, 21f, and 24

\begin{tabular}{ccccccc}
$\begin{array}{c}\text { amino } \\
\text { acid }\end{array}$ & $\begin{array}{c}\lambda_{\text {Abs }} \\
(\mathrm{nm})^{a}\end{array}$ & $\varepsilon\left(\mathrm{cm}^{-1} \mathrm{M}^{-1}\right)$ & $\begin{array}{c}\lambda_{\text {Em }} \\
(\mathrm{nm})^{a}\end{array}$ & $\begin{array}{c}\text { Stokes } \\
\text { shift } \\
(\mathrm{nm})\end{array}$ & QY & $\begin{array}{c}\text { brightness } \\
\left(\mathrm{cm}^{-1} \mathrm{M}^{-1}\right)\end{array}$ \\
\hline 21a & 256 & 23034 & 418 & 162 & 0.17 & 3857 \\
21b & 258 & 18504 & 454 & 196 & 0.02 & 350 \\
21c & 285 & 19212 & 384 & 99 & 0.004 & 82 \\
21e & 286 & 10309 & 417 & 131 & 0.18 & 1866 \\
21f & 257 & 19903 & 384 & 127 & 0.03 & 533 \\
24 & 283 & 9516 & 432 & 149 & 0.09 & 856
\end{tabular}

${ }^{a}$ Spectra were recorded in methanol at: $1 \times 10^{-5} \mathrm{M}$.

chromophores allows vibration relaxation in the excited and ground states, resulting in very large Stokes shifts. $\alpha$-Amino acids 21a and 21e were also shown to have good quantum yields of 17 and $18 \%$, respectively, and consequently, the highest brightness values.

As the $p$-methoxyphenyl-substituted benzotriazole 21a showed a number of favorable photophysical properties and was found to be the brightest $\alpha$-amino acid, it was decided to further explore the potential applications of this compound with a solvatochromic study. As expected, the absorption maxima $(256 \mathrm{~nm})$ in tetrahydrofuran (THF), methanol, and phosphate-buffered saline (PBS) were found to be independent of polarity (Figure 3a), indicating negligible intramolecular interactions between the electron-rich methoxyphenyl and electron-deficient benzotriazole moieties in the ground state. In contrast, the emission spectra were found to be highly dependent on the solvent used, with increasing polarity leading to broadened structureless emission spectra at longer wavelengths (Figure $3 \mathrm{~b}) .^{24}$ For example, in THF, an emission maximum at $372 \mathrm{~nm}$ was observed, while in PBS, the emission maximum was found to be at $449 \mathrm{~nm}$. The bathochromic shift of 21a is indicative of greater stabilization of the highly polar, (a)

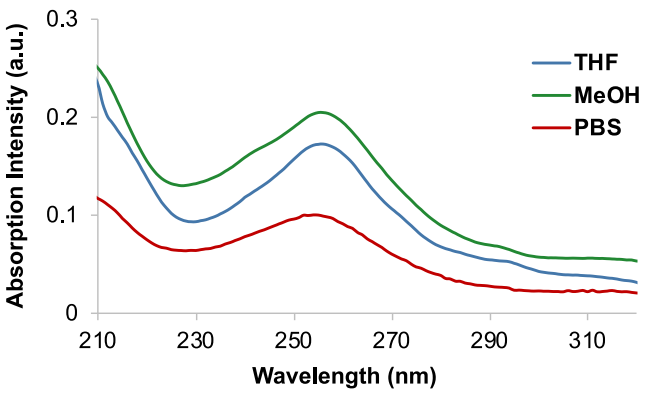

(b)

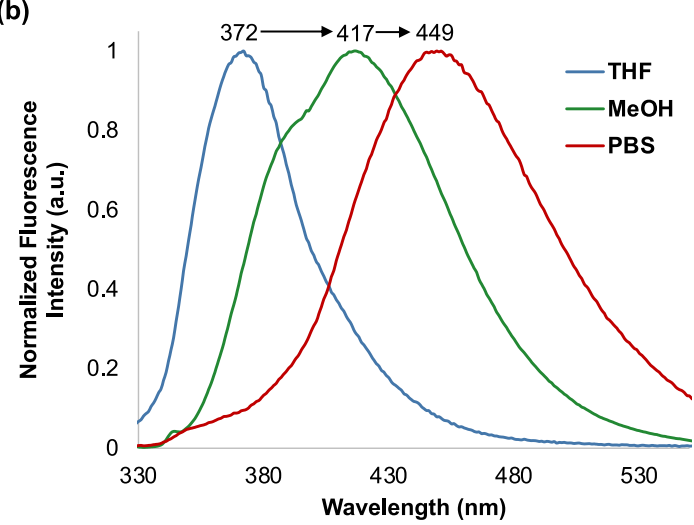

Figure 3. Absorption and emission spectra of 21a in various solvents. All spectra were recorded using a concentration of $1 \times 10^{-5} \mathrm{M}$.

excited state by the reorganization and relaxation of the dipoles of the increasingly polar solvent molecules. The broadened emission spectra of $21 \mathrm{a}$ in more polar solvents are due to a combination of locally excited and internal charge transfer states. $^{25}$ These two components can be observed in the emission spectrum in methanol, where the shoulder peak and the main band at longer wavelength are due to the locally excited and internal charge transfer states, respectively.

\section{CONCLUSIONS}

In summary, a new class of heterocycle-containing $\alpha$-amino acid has been synthesized using a nucleophilic aromatic substitution reaction of 2-fluoronitrobenzenes with a 3aminoalanine derivative and a one-pot diazo activation and cyclization process using a polymer-supported nitrite reagent under mild conditions. The structural diversity of these compounds was increased by Suzuki-Miyaura cross-coupling reactions with bromine-substituted benzotriazoles. Investigation of the photophysical properties of the resulting $\alpha$-amino acids with extended conjugation showed that benzotriazoles with electron-rich aryl substituents were strongly fluorescent in the visible region and displayed MegaStokes shifts. The environment-sensitive nature of these compounds was also demonstrated with a solvatochromic study of $\alpha$-amino acid 21a, which showed a bathochromic shift in more polar solvents. Future work will explore an expansion of the structural diversity of these benzotriazole-derived $\alpha$-amino acids and investigate their potential for biological imaging.

\section{EXPERIMENTAL SECTION}

The synthesis of $\mathbf{1 3}$ and $\mathbf{1 4}$ has been previously described in the literature. ${ }^{16,17}$ All reagents and starting materials were obtained from commercial sources and used as received unless otherwise stated. Dry solvents were purified using a solvent purification system. Brine refers 
to a saturated solution of sodium chloride. All reactions were performed in oven-dried glassware under an atmosphere of argon unless otherwise stated. Flash column chromatography was carried out using silica gel $(40-63 \mu \mathrm{m})$ and neutral aluminium oxide (50$200 \mu \mathrm{m})$. Aluminium-backed plates precoated with silica gel 60 $\left(\mathrm{UV}_{254}\right)$ were used for thin layer chromatography and were visualized under ultraviolet light and by staining with $\mathrm{KMnO}_{4}$, ninhydrin or vanillin. ${ }^{1} \mathrm{H}$ NMR spectra were recorded on an NMR spectrometer at 400 or $500 \mathrm{MHz}$, and data are reported as follows: chemical shift in $\mathrm{ppm}$ relative to tetramethylsilane or the solvent as the internal standard $\left(\mathrm{CDCl}_{3}, \delta 7.26 \mathrm{ppm}\right)$, multiplicity ( $\mathrm{s}=$ singlet, $\mathrm{d}=$ doublet, $\mathrm{t}$ $=$ triplet, $\mathrm{q}=$ quartet, $\mathrm{m}=$ multiplet or overlap of nonequivalent resonances, integration). ${ }^{13} \mathrm{C}\left\{{ }^{1} \mathrm{H}\right\}$ NMR spectra were recorded on an NMR spectrometer at 101 or $126 \mathrm{MHz}$, and data are reported as follows: chemical shift in ppm relative to tetramethylsilane or the solvent as internal standard $\left(\mathrm{CDCl}_{3}, \delta 77.0 \mathrm{ppm}\right)$, multiplicity with respect to hydrogen (deduced from DEPT experiments, $\mathrm{C}, \mathrm{CH}, \mathrm{CH}_{2}$ or $\mathrm{CH}_{3}$ ). IR spectra were recorded on a FTIR spectrometer; wavenumbers are indicated in $\mathrm{cm}^{-1}$. Mass spectra were recorded using electron impact or electrospray ionization techniques. HRMS spectra were recorded using a dual-focusing magnetic analyzer mass spectrometer. Melting points are uncorrected. Optical rotations were determined as solutions irradiating with the sodium $\mathrm{D}$ line $(\lambda$ $=589 \mathrm{~nm})$ using a polarimeter. $[\alpha]_{\mathrm{D}}$ values are given in units $10^{-1} \mathrm{deg}$ $\mathrm{cm}^{2} \mathrm{~g}^{-1}$. Fluorescence spectra were recorded on a spectrofluorophotometer. Emission data were measured using an excitation slit width of $3 \mathrm{~nm}$ and emission slit width of $3 \mathrm{~nm}$. Quantum yield data were measured using anthracene and L-tryptophan as standard references.

Methyl (2S)-2-[(Benzyloxycarbonyl)amino]-3-[(2' nitrophenyl)amino]propanoate (15a). To a solution of methyl (2S)-3-amino-2-[(benzyloxycarbonyl)amino]propanoate hydrochloride (14) $(0.250 \mathrm{~g}, 0.991 \mathrm{mmol})$ in acetonitrile $(7.5 \mathrm{~mL})$ under argon were added 2-fluoronitrobenzene $(0.310 \mathrm{~mL}, 2.97 \mathrm{mmol})$ and triethylamine $(0.410 \mathrm{~mL}, 2.97 \mathrm{mmol})$, and the reaction mixture was stirred under reflux for $16 \mathrm{~h}$. After cooling the reaction to ambient temperature, the solvent was removed in vacuo. The resulting residue was dissolved in ethyl acetate $(50 \mathrm{~mL})$, washed with water $(3 \times 50$ $\mathrm{mL})$, and brine $(50 \mathrm{~mL})$. The organic layer was dried $\left(\mathrm{MgSO}_{4}\right)$, filtered, and concentrated in vacuo. Purification by flash column chromatography eluting with $0-20 \%$ ethyl acetate in dichloromethane gave methyl (2S)-2-[(benzyloxycarbonyl)amino]-3-[( $2^{\prime}$ nitrophenyl)amino $]$ propanoate $(\mathbf{1 5 a})$ as a yellow solid $(0.330 \mathrm{~g}$, $89 \%$ ). mp $82-84{ }^{\circ} \mathrm{C}$; IR (neat) $3348,2956,1721,1620,1512,1265$, 1234, $741 \mathrm{~cm}^{-1}$; $[\alpha]_{\mathrm{D}}^{25}+50.8\left(\right.$ c $\left.0.3, \mathrm{CHCl}_{3}\right) ;{ }^{1} \mathrm{H}$ NMR $(400 \mathrm{MHz}$, $\left.\mathrm{CDCl}_{3}\right): \delta 3.64-3.74(\mathrm{~m}, 5 \mathrm{H}), 4.57(\mathrm{dt}, J=6.7,5.7 \mathrm{~Hz}, 1 \mathrm{H}), 5.04(\mathrm{~s}$, $2 \mathrm{H}), 5.63(\mathrm{~d}, J=6.7 \mathrm{~Hz}, 1 \mathrm{H}), 6.69(\mathrm{br} \mathrm{t}, J=8.6 \mathrm{~Hz}, 1 \mathrm{H}), 6.98(\mathrm{~d}, J=$ $8.6 \mathrm{~Hz}, 1 \mathrm{H}), 7.27-7.51(\mathrm{~m}, 6 \mathrm{H}), 8.16(\mathrm{dd}, J=8.6,1.2 \mathrm{~Hz}, 1 \mathrm{H}), 8.22$ $(\mathrm{t}, J=5.6 \mathrm{~Hz}, 1 \mathrm{H}) ;{ }^{13} \mathrm{C}\left\{{ }^{1} \mathrm{H}\right\} \operatorname{NMR}\left(101 \mathrm{MHz}, \mathrm{CDCl}_{3}\right): \delta 44.6$ $\left(\mathrm{CH}_{2}\right), 53.1\left(\mathrm{CH}_{3}\right), 53.6(\mathrm{CH}), 67.4\left(\mathrm{CH}_{2}\right), 113.7(\mathrm{CH}), 116.3$ $(\mathrm{CH}), 127.0(\mathrm{CH}), 128.3(2 \times \mathrm{CH}), 128.4(\mathrm{CH}), 128.7(2 \times \mathrm{CH})$, 132.8 (C), 136.0 (C), $136.4(\mathrm{CH}), 144.9(\mathrm{C}), 155.9$ (C), 170.8 (C); MS (ESI) $m / z: 396\left(\mathrm{M}+\mathrm{Na}^{+}, 100\right)$; HRMS (ESI): calcd for $\mathrm{C}_{18} \mathrm{H}_{19} \mathrm{~N}_{3} \mathrm{NaO}_{6}\left(\mathrm{M}+\mathrm{Na}^{+}\right)$, 396.1166; found, 396.1158 .

Methyl (2S)-2-(Benzyloxycarbonyl)amino]-3-[(5'-methoxy$\mathbf{2}^{\prime}$-nitrophenyl)amino]propanoate (15b). Methyl (2S)-2(benzyloxycarbonyl)amino]-3-[(5'-methoxy-2'-nitrophenyl)amino]propanoate (15b) was synthesized as described for methyl (2S)-2[(benzyloxycarbonyl)amino]-3-[(2'-nitrophenyl)amino]propanoate (15a) using methyl (2S)-3-amino-2-[(benzyloxycarbonyl)amino]propanoate hydrochloride (14) $(0.200 \mathrm{~g}, 0.793 \mathrm{mmol})$, 3-fluoro-4nitroanisole $(0.407 \mathrm{~g}, 2.38 \mathrm{mmol})$, and triethylamine $(0.332 \mathrm{~mL}, 2.38$ $\mathrm{mmol})$ in acetonitrile $(6.0 \mathrm{~mL})$. Purification by flash column chromatography eluting with $0-20 \%$ ethyl acetate in dichloromethane gave methyl (2S)-2-(benzyloxycarbonyl)amino]-3-[(5'methoxy-2'-nitrophenyl)amino]propanoate $(\mathbf{1 5 b})$ as a yellow solid (0.277 g, 87\%). mp 82-84 ${ }^{\circ} \mathrm{C}$; IR (neat) $3356,2955,1713,1620$, $1582,1497,1226,1221,748 \mathrm{~cm}^{-1} ;[\alpha]_{\mathrm{D}}^{23}+20.7\left(c 1.0, \mathrm{CHCl}_{3}\right) ;{ }^{1} \mathrm{H}$ NMR $\left(400 \mathrm{MHz}, \mathrm{CDCl}_{3}\right): \delta 3.73$ (ddd, $\left.J=13.7,6.2,5.7 \mathrm{~Hz}, 1 \mathrm{H}\right)$, 3.77 (ddd, $J=13.7,6.2,5.7 \mathrm{~Hz}, 1 \mathrm{H}), 3.80(\mathrm{~s}, 3 \mathrm{H}), 3.87(\mathrm{~s}, 3 \mathrm{H}), 4.64$ $(\mathrm{dt}, J=6.9,6.2 \mathrm{~Hz}, 1 \mathrm{H}), 5.13(\mathrm{~s}, 2 \mathrm{H}), 5.63(\mathrm{~d}, J=6.9 \mathrm{~Hz}, 1 \mathrm{H}), 6.27$ $(\mathrm{dd}, J=9.5,2.5 \mathrm{~Hz}, 1 \mathrm{H}), 6.43(\mathrm{~d}, J=2.5 \mathrm{~Hz}, 1 \mathrm{H}), 7.28-7.42(\mathrm{~m}$, $5 \mathrm{H}), 8.14(\mathrm{~d}, J=9.5 \mathrm{~Hz}, 1 \mathrm{H}), 8.52(\mathrm{t}, J=5.7 \mathrm{~Hz}, 1 \mathrm{H}) ;{ }^{13} \mathrm{C}\left\{{ }^{1} \mathrm{H}\right\}$ NMR $\left(101 \mathrm{MHz}, \mathrm{CDCl}_{3}\right): \delta 44.6\left(\mathrm{CH}_{2}\right), 53.1\left(\mathrm{CH}_{3}\right), 53.2(\mathrm{CH})$, $55.9\left(\mathrm{CH}_{3}\right), 67.3\left(\mathrm{CH}_{2}\right), 95.4(\mathrm{CH}), 105.6(\mathrm{CH}), 127.0(\mathrm{C}), 128.2$ $(2 \times \mathrm{CH}), 128.4(\mathrm{CH}), 128.6(2 \times \mathrm{CH}), 129.3(\mathrm{CH}), 135.9(\mathrm{C})$, 147.3 (C), 155.7 (C), 166.2 (C), 170.7 (C); MS (ESI) $m / z: 426$ (M $\left.+\mathrm{Na}^{+}, 100\right)$; HRMS (ESI): calcd for $\mathrm{C}_{19} \mathrm{H}_{21} \mathrm{~N}_{3} \mathrm{NaO}_{7}\left(\mathrm{M}+\mathrm{Na}^{+}\right)$, 426.1272; found, 426.1266 .

Methyl (2S)-2-(Benzyloxycarbonyl)amino]-3-[(4'-bromo-2' nitrophenyl)amino]propanoate (15c). Methyl (2S)-2(benzyloxycarbonyl)amino]-3-[(4'-bromo-2'-nitrophenyl)amino]propanoate (15c) was synthesized as described for methyl (2S)-2[(benzyloxycarbonyl)amino]-3-[(2'-nitrophenyl)amino]propanoate (15a) using methyl (2S)-3-amino-2-[(benzyloxycarbonyl)amino]propanoate hydrochloride (14) (3.97 g, $13.8 \mathrm{mmol})$, 5-bromo-2fluoro-1-nitrobenzene $(5.10 \mathrm{~mL}, 41.3 \mathrm{mmol})$, and triethylamine $(5.90$ $\mathrm{mL}, 41.3 \mathrm{mmol})$ in acetonitrile $(50 \mathrm{~mL})$. Purification by flash column chromatography eluting with $0-20 \%$ ethyl acetate in dichloromethane gave methyl (2S)-2-(benzyloxycarbonyl)amino]-3-[(4'bromo-2'-nitrophenyl)amino]propanoate (15c) as a yellow solid (5.75 g, 92\%). mp 86-89 ${ }^{\circ} \mathrm{C}$; IR (neat) $3364,2955,1721,1612$, 1504, 1227, $1065 \mathrm{~cm}^{-1}$; $[\alpha]_{\mathrm{D}}^{25}+14.3\left(c\right.$ 1.1, $\left.\mathrm{CHCl}_{3}\right) ;{ }^{1} \mathrm{H}$ NMR (400 $\left.\mathrm{MHz}, \mathrm{CDCl}_{3}\right): \delta 3.60-3.76(\mathrm{~m}, 5 \mathrm{H}), 4.60(\mathrm{dt}, J=6.9,5.8 \mathrm{~Hz}, 1 \mathrm{H})$, $5.06(\mathrm{~d}, J=12.2 \mathrm{~Hz}, 1 \mathrm{H}), 5.11(\mathrm{~d}, J=12.2 \mathrm{~Hz}, 1 \mathrm{H}), 6.06(\mathrm{~d}, J=6.9$ $\mathrm{Hz}, 1 \mathrm{H}), 6.81(\mathrm{~d}, J=9.1 \mathrm{~Hz}, 1 \mathrm{H}), 7.20-7.30(\mathrm{~m}, 5 \mathrm{H}), 7.36(\mathrm{dd}, J=$ 9.1, $1.5 \mathrm{~Hz}, 1 \mathrm{H}), 8.16(\mathrm{~d}, J=1.5 \mathrm{~Hz}, 1 \mathrm{H}), 8.19(\mathrm{t}, J=6.0 \mathrm{~Hz}, 1 \mathrm{H})$; ${ }^{13} \mathrm{C}\left\{{ }^{1} \mathrm{H}\right\}$ NMR $\left(101 \mathrm{MHz}, \mathrm{CDCl}_{3}\right): \delta 44.2\left(\mathrm{CH}_{2}\right), 52.8\left(\mathrm{CH}_{3}\right), 53.2$ $(\mathrm{CH}), 67.0\left(\mathrm{CH}_{2}\right), 107.0(\mathrm{C}), 115.3(\mathrm{CH}), 127.9(2 \times \mathrm{CH}), 128.1$ $(\mathrm{CH}), 128.3(2 \times \mathrm{CH}), 128.6(\mathrm{CH}), 132.5(\mathrm{C}), 135.8(\mathrm{C}), 138.7$ $(\mathrm{CH}), 143.6$ (C), 155.9 (C), 170.4 (C); MS (ESI) $m / z: 474(\mathrm{M}+$ $\left.\mathrm{Na}^{+}, 100\right)$; HRMS (ESI): calcd for $\mathrm{C}_{18} \mathrm{H}_{18}{ }^{79} \mathrm{BrN}_{3} \mathrm{NaO}_{6}\left(\mathrm{M}+\mathrm{Na}^{+}\right)$, 474.0271; found, 474.0276.

Methyl (2S)-2-(Benzyloxycarbonyl)amino]-3-\{[2' -nitro-4' (trifluoromethyl)phenyl]amino\}propanoate (15d). Methyl (2S)-2-(benzyloxycarbonyl)amino $]-3-\left\{\left[2^{\prime}\right.\right.$-nitro-4' -(trifluoromethyl)phenyl]amino propanoate (15d) was synthesized as described for methyl (2S)-2-[(benzyloxycarbonyl)amino $]-3-\left[\left(2^{\prime}\right.\right.$-nitrophenyl)amino]propanoate (15a) using methyl (2S)-3-amino-2[(benzyloxycarbonyl)amino]propanoate hydrochloride (14) $(0.200$ g, $0.793 \mathrm{mmol}$ ), 2-fluoro-5-(trifluoromethyl)-1-nitrobenzene (0.333 $\mathrm{mL}, 2.38 \mathrm{mmol})$, and triethylamine $(0.332 \mathrm{~mL}, 2.38 \mathrm{mmol})$ in acetonitrile $(6.0 \mathrm{~mL})$. Purification by flash column chromatography eluting with $0-20 \%$ ethyl acetate in dichloromethane gave methyl (2S)-2-(benzyloxycarbonyl)amino]-3-\{[2'-nitro-4' -(trifluoromethyl)phenyl] amino propanoate $(15 \mathrm{~d})$ as a yellow solid $(0.295 \mathrm{~g}, 84 \%) \mathrm{mp}$ $84-86^{\circ} \mathrm{C}$; IR (neat) $3356,2955,1713,1636,1535,1435,1319$, 1227, 1111, 756, $694 \mathrm{~cm}^{-1} ;[\alpha]_{\mathrm{D}}^{23}+25.9\left(\mathrm{c} 1.0, \mathrm{CHCl}_{3}\right) ;{ }^{1} \mathrm{H} \mathrm{NMR}$ $\left(500 \mathrm{MHz}, \mathrm{CDCl}_{3}\right): \delta 3.75-3.82(\mathrm{~m}, 4 \mathrm{H}), 3.85$ (ddd, $J=13.8,5.8$, $5.4 \mathrm{~Hz}, 1 \mathrm{H}), 4.65(\mathrm{dt}, J=6.9,5.4 \mathrm{~Hz}, 1 \mathrm{H}), 5.09(\mathrm{~d}, J=12.1 \mathrm{~Hz}, 1 \mathrm{H})$, $5.14(\mathrm{~d}, J=12.1 \mathrm{~Hz}, 1 \mathrm{H}), 5.83(\mathrm{~d}, J=6.9 \mathrm{~Hz}, 1 \mathrm{H}), 7.08(\mathrm{~d}, J=9.0$ $\mathrm{Hz}, 1 \mathrm{H}), 7.27-7.43(\mathrm{~m}, 5 \mathrm{H}), 7.56(\mathrm{~d}, J=9.0 \mathrm{~Hz}, 1 \mathrm{H}), 8.42(\mathrm{~s}, 1 \mathrm{H})$, $8.48(\mathrm{t}, J=5.8 \mathrm{~Hz}, 1 \mathrm{H}) ;{ }^{13} \mathrm{C}\left\{{ }^{1} \mathrm{H}\right\}$ NMR $\left(126 \mathrm{MHz}, \mathrm{CDCl}_{3}\right): \delta 44.5$ $\left(\mathrm{CH}_{2}\right), 53.2\left(\mathrm{CH}_{3}\right), 53.5(\mathrm{CH}), 67.4\left(\mathrm{CH}_{2}\right), 114.5(\mathrm{CH}), 118.2(\mathrm{C}$, $\left.\mathrm{q},{ }^{2} J_{\mathrm{CF}}=34.4 \mathrm{~Hz}\right), 123.5\left(\mathrm{C}, \mathrm{q},{ }_{\mathrm{CF}}=271.0 \mathrm{~Hz}\right), 125.0\left(\mathrm{CH}, \mathrm{q},{ }^{3} J_{\mathrm{CF}}=\right.$ $4.3 \mathrm{~Hz}), 128.3(2 \times \mathrm{CH}), 128.4(\mathrm{CH}), 128.6(2 \times \mathrm{CH}), 131.6(\mathrm{C})$, $132.3\left(\mathrm{CH}, \mathrm{q},{ }^{3} J_{\mathrm{CF}}=3.2 \mathrm{~Hz}\right), 135.9(\mathrm{C}), 146.6(\mathrm{C}), 156.0(\mathrm{C}), 170.4$ (C); MS (ESI) $m / z: 464\left(\mathrm{M}+\mathrm{Na}^{+}, 100\right)$; HRMS (ESI): calcd for $\mathrm{C}_{19} \mathrm{H}_{18} \mathrm{~F}_{3} \mathrm{~N}_{3} \mathrm{NaO}_{6}\left(\mathrm{M}+\mathrm{Na}^{+}\right)$, 464.1040; found, 464.1040.

Methyl (2S)-2-(Benzyloxycarbonyl)amino $]-3-\left[\left(4^{\prime}, 5^{\prime}-\right.\right.$ dichloro-2'-nitrophenyl)amino]propanoate (15e). Methyl (2S)2-(benzyloxycarbonyl)amino]-3-[(4',5'-dichloro-2'-nitrophenyl)amino]propanoate $(\mathbf{1 5 e})$ was synthesized as described for methyl $(2 S)-2$-[(benzyloxycarbonyl)amino]-3-[(2'-nitrophenyl)amino]propanoate (15a) using methyl (2S)-3-amino-2[(benzyloxycarbonyl)amino]propanoate hydrochloride (14) (0.400 g, $1.59 \mathrm{mmol})$, 2-fluoro-4,5-dichloro-1-nitrobenzene $(0.628 \mathrm{~mL}, 4.77$ $\mathrm{mmol})$, and triethylamine $(0.665 \mathrm{~mL}, 4.77 \mathrm{mmol})$ in acetonitrile $(12$ $\mathrm{mL}$ ). Purification by flash column chromatography eluting with $0-$ $20 \%$ ethyl acetate in dichloromethane gave methyl (2S)-2(benzyloxycarbonyl)amino $]-3-\left[\left(4^{\prime}, 5^{\prime}\right.\right.$-dichloro-2' -nitrophenyl)- 
amino]propanoate (15e) as a yellow solid $(0.599 \mathrm{~g}, 85 \%)$. mp 94-96 ${ }^{\circ} \mathrm{C}$; IR (neat) 3358, 2953, 1717, 1612, 1521, 1485, $1223 \mathrm{~cm}^{-1}$; $[\alpha]_{\mathrm{D}}^{23}$ $+28.4\left(\right.$ c 1.0, $\left.\mathrm{CHCl}_{3}\right) ;{ }^{1} \mathrm{H}$ NMR $\left(400 \mathrm{MHz}, \mathrm{CDCl}_{3}\right): \delta 3.72$ (ddd, $J=$ 13.6, 5.6, $5.0 \mathrm{~Hz}, 1 \mathrm{H}), 3.76(\mathrm{ddd}, J=13.6,5.6,5.0 \mathrm{~Hz}, 1 \mathrm{H}), 3.81(\mathrm{~s}$, $3 \mathrm{H}), 4.64(\mathrm{dt}, J=6.1,5.6 \mathrm{~Hz}, 1 \mathrm{H}), 5.13(\mathrm{~s}, 2 \mathrm{H}), 5.71(\mathrm{~d}, J=6.1 \mathrm{~Hz}$, $1 \mathrm{H}), 7.13(\mathrm{~s}, 1 \mathrm{H}), 7.27-7.44(\mathrm{~m}, 5 \mathrm{H}), 8.18(\mathrm{t}, J=5.0 \mathrm{~Hz}, 1 \mathrm{H}), 8.26$ (s, $1 \mathrm{H}) ;{ }^{13} \mathrm{C}\left\{{ }^{1} \mathrm{H}\right\}$ NMR $\left(101 \mathrm{MHz}, \mathrm{CDCl}_{3}\right): \delta 44.8\left(\mathrm{CH}_{2}\right), 53.2$ $\left(\mathrm{CH}_{3}\right), 53.4(\mathrm{CH}), 67.5\left(\mathrm{CH}_{2}\right), 115.1(\mathrm{CH}), 119.7(\mathrm{C}), 127.9(\mathrm{CH})$, $128.2(2 \times \mathrm{CH}), 128.4(\mathrm{CH}), 128.6(2 \times \mathrm{CH}), 131.2(\mathrm{C}), 135.7(\mathrm{C})$, 141.3 (C), 143.6 (C), 155.9 (C), 170.3 (C); MS (ESI) $m / z: 464$ (M $\left.+\mathrm{Na}^{+}, 100\right)$; HRMS (ESI): calcd for $\mathrm{C}_{18} \mathrm{H}_{17}{ }^{35} \mathrm{Cl}_{2} \mathrm{~N}_{3} \mathrm{NaO}_{6}\left(\mathrm{M}+\mathrm{Na}^{+}\right)$, 464.0387; found, 464.0373 .

Methyl (2S)-2-(Benzyloxycarbonyl)amino]-3-[(5'-chloro-4' methyl-2'-nitrophenyl)amino]propanoate (15f). Methyl (2S)2-(benzyloxycarbonyl) amino $]-3$ - [ ( $5^{\prime}$ - chloro- $4^{\prime}$ - methyl-2' nitrophenyl)amino]propanoate (15f) was synthesized as described for methyl (2S)-2-[(benzyloxycarbonyl)amino]-3-[(2'-nitrophenyl)amino]propanoate (15a) using methyl (2S)-3-amino-2[(benzyloxycarbonyl)amino] propanoate hydrochloride (14) (0.400 g, $1.59 \mathrm{mmol})$, 1-chloro-5-fluoro-2-methyl-4-nitrobenzene $(0.904 \mathrm{~g}$, $4.77 \mathrm{mmol})$, and triethylamine $(0.665 \mathrm{~mL}, 4.77 \mathrm{mmol})$ in acetonitrile $(12 \mathrm{~mL})$. Purification by flash column chromatography eluting with $0-20 \%$ ethyl acetate in dichloromethane gave methyl (2S)-2(benzyloxycarbonyl)amino]-3-[(5'-chloro-4'-methyl-2'-nitrophenyl)amino]propanoate (15f) as a yellow oil $(0.643 \mathrm{~g}, 96 \%)$. IR (neat) $3360,2951,1738,1726,1626,1501,1228,1217,978 \mathrm{~cm}^{-1} ;[\alpha]_{\mathrm{D}}^{23}$ +23.5 (c 1.0, $\left.\mathrm{CHCl}_{3}\right) ;{ }^{1} \mathrm{H}$ NMR $\left(400 \mathrm{MHz}, \mathrm{CDCl}_{3}\right): \delta 2.28(\mathrm{~s}, 3 \mathrm{H})$, 3.71 (ddd, $J=13.6,5.7,5.3 \mathrm{~Hz}, 1 \mathrm{H}), 3.75$ (ddd, $J=13.6,5.7,5.3 \mathrm{~Hz}$, $1 \mathrm{H}), 3.80(\mathrm{~s}, 3 \mathrm{H}), 4.65(\mathrm{dt}, J=6.3,5.3 \mathrm{~Hz}, 1 \mathrm{H}), 5.13(\mathrm{~s}, 2 \mathrm{H}), 5.70$ $(\mathrm{d}, J=6.3 \mathrm{~Hz}, 1 \mathrm{H}), 7.00(\mathrm{~s}, 1 \mathrm{H}), 7.27-7.39(\mathrm{~m}, 5 \mathrm{H}), 8.03(\mathrm{~s}, 1 \mathrm{H})$, $8.08(\mathrm{t}, J=5.7 \mathrm{~Hz}, 1 \mathrm{H}) ;{ }^{13} \mathrm{C}\left\{{ }^{1} \mathrm{H}\right\}$ NMR $\left(101 \mathrm{MHz}, \mathrm{CDCl}_{3}\right): \delta 18.9$ $\left(\mathrm{CH}_{3}\right), 44.7\left(\mathrm{CH}_{2}\right), 53.1\left(\mathrm{CH}_{3}\right), 53.5(\mathrm{CH}), 67.4\left(\mathrm{CH}_{2}\right), 113.8$ $(\mathrm{CH}), 124.2(\mathrm{C}), 128.1(\mathrm{CH}), 128.2(2 \times \mathrm{CH}), 128.3(\mathrm{CH}), 128.6$ $(2 \times \mathrm{CH}), 131.0(\mathrm{C}), 135.9$ (C), 143.6 (C), 143.6 (C), 155.9 (C), 170.5 (C); MS (ESI) $m / z: 444\left(\mathrm{M}+\mathrm{Na}^{+}, 100\right)$; HRMS (ESI): calcd for $\mathrm{C}_{19} \mathrm{H}_{20}{ }^{35} \mathrm{ClN}_{3} \mathrm{NaO}_{6}\left(\mathrm{M}+\mathrm{Na}^{+}\right)$, 444.0933; found, 444.0916.

General Procedure for the Preparation of PolymerSupported Nitrite. ${ }^{13 a}$ The polymer-supported nitrite reagent was prepared by the addition of Amberlyst A26 hydroxide form resin $(10.0 \mathrm{~g}, 40.0 \mathrm{mmol})$ to a solution of sodium nitrite $(5.50 \mathrm{~g}, 80.0$ $\mathrm{mmol})$ in water $(200 \mathrm{~mL})$. The mixture was stirred at room temperature for $0.5 \mathrm{~h}$. The polymer-supported nitrite was filtered and washed with water until the $\mathrm{pH}$ of the filtrate became neutral. The content of the polymer-supported nitrite was $3.5 \mathrm{mmol}$ of $\mathrm{NO}_{2}{ }^{-}$per g. ${ }^{13 a}$

Methyl (2S)-2-[(Benzyloxycarbonyl)amino]-3-(1H-benzo[d][1.2.3]triazol-1'-yl)propanoate (17a). To a solution of (2S)-2[(benzyloxycarbonyl)amino]-3-[(2'-nitrophenyl)amino]propanoate (15a) $(0.320 \mathrm{~g}, 0.857 \mathrm{mmol})$ in methanol $(12 \mathrm{~mL})$ was added tin(II) dichloride dihydrate $(0.970 \mathrm{~g}, 4.29 \mathrm{mmol})$, and the reaction mixture was stirred under reflux for $20 \mathrm{~h}$. After cooling the reaction to ambient temperature, the solvent was removed in vacuo, and the resulting residue dissolved in ethyl acetate $(50 \mathrm{~mL})$ and mixed with a saturated solution of aqueous sodium hydrogen carbonate $(30 \mathrm{~mL})$. The biphasic mixture was filtered through a pad of Celite and the organic layer separated from the aqueous layer. The product was further extracted from the aqueous layer with ethyl acetate $(2 \times 30 \mathrm{~mL})$, and the combined organic layers were washed with brine $(50 \mathrm{~mL})$, dried $\left(\mathrm{MgSO}_{4}\right)$, filtered, and concentrated in vacuo. Purification by flash column chromatography eluting with $10 \%$ ethyl acetate in dichloromethane gave methyl (2S)-2-[(benzyloxycarbonyl)amino]-3-[(2'aminophenyl)amino]propanoate (16a) as a white solid $(0.244 \mathrm{~g}$, $83 \%)$. This material was then used immediately in the following step. To a solution of methyl (2S)-2-[(benzyloxycarbonyl)amino]-3-[(2'aminophenyl)amino]propanoate (16a) $(0.244 \mathrm{~g}, 0.711 \mathrm{mmol})$ in acetonitrile $(12 \mathrm{~mL})$ at $-10{ }^{\circ} \mathrm{C}$ was added $p$-toluenesulfonic acid (0.405 g, $2.13 \mathrm{mmol})$ and polymer-supported nitrite (0.609 g containing $2.13 \mathrm{mmol}$ of $\mathrm{NO}_{2}^{-}$), and the reaction mixture was stirred at this temperature for $3 \mathrm{~h}$. The reaction mixture was filtered, and the resin was washed with dichloromethane $(50 \mathrm{~mL})$. The organic layers were washed with a saturated solution of aqueous sodium hydrogen carbonate $(50 \mathrm{~mL})$ and brine $(50 \mathrm{~mL})$, dried $\left(\mathrm{MgSO}_{4}\right)$, filtered, and concentrated in vacuo. Purification by flash column chromatography eluting with $10 \%$ ethyl acetate in dichloromethane gave methyl $(2 S)$ 2-[(benzyloxycarbonyl)amino]-3-( $1 H$-benzo[d][1.2.3]triazol-1'-yl)propanoate $(17 \mathrm{a})$ as a white solid $(0.148 \mathrm{~g}, 59 \%) . \mathrm{mp} 82-85^{\circ} \mathrm{C}$; IR (neat) $3325,2955,1713,1504,1211,1057,741 \mathrm{~cm}^{-1} ;[\alpha]_{\mathrm{D}}^{25}+37.0(c$ $\left.0.4, \mathrm{CHCl}_{3}\right) ;{ }^{1} \mathrm{H}$ NMR $\left(400 \mathrm{MHz}, \mathrm{CDCl}_{3}\right): \delta 3.75(\mathrm{~s}, 3 \mathrm{H}), 4.88(\mathrm{dt}, J$ $=7.0,4.4 \mathrm{~Hz}, 1 \mathrm{H}), 5.05-5.18(\mathrm{~m}, 4 \mathrm{H}), 5.66(\mathrm{~d}, J=7.0 \mathrm{~Hz}, 1 \mathrm{H})$, 7.29-7.45 (m, 8H), $8.03(\mathrm{br} \mathrm{d}, J=8.0 \mathrm{~Hz}, 1 \mathrm{H}) ;{ }^{13} \mathrm{C}\left\{{ }^{1} \mathrm{H}\right\}$ NMR $(101$ $\left.\mathrm{MHz}, \mathrm{CDCl}_{3}\right): \delta 48.7\left(\mathrm{CH}_{2}\right), 53.3\left(\mathrm{CH}_{3}\right), 54.4(\mathrm{CH}), 67.4\left(\mathrm{CH}_{2}\right)$, 109.2 (CH), $120.2(\mathrm{CH}), 124.2(\mathrm{CH}), 127.9(\mathrm{CH}), 128.3(2 \times \mathrm{CH})$, $128.5(\mathrm{CH}), 128.7(2 \times \mathrm{CH}), 133.9(\mathrm{C}), 136.0(\mathrm{C}), 145.8(\mathrm{C}), 155.8$ (C), 169.5 (C); MS (ESI) $m / z: 377\left(\mathrm{M}+\mathrm{Na}^{+}, 100\right)$; HRMS (ESI): calcd for $\mathrm{C}_{18} \mathrm{H}_{18} \mathrm{~N}_{4} \mathrm{NaO}_{4}\left(\mathrm{M}+\mathrm{Na}^{+}\right)$, 377.1220; found, 377.1223.

Methyl (2S)-2-[(Benzyloxycarbonyl)amino]-3-(6'-methoxy1H-benzo[d][1.2.3]triazol-1'-yl)propanoate (17b). Methyl (2S)2 -[(benzyloxycarbonyl)amino]-3-(6'-methoxy-1H-benzo[d][1.2.3]triazol-1'-yl)propanoate (17b) was synthesized as described for methyl (2S)-2-[(benzyloxycarbonyl)amino]-3-(1H-benzo[d][1.2.3]triazol-1'-yl)propanoate (17a) using methyl (2S)-2(benzyloxycarbonyl)amino]-3-[( $5^{\prime}$-methoxy-2' -nitrophenyl)amino]propanoate $(15 \mathrm{~b})(0.270 \mathrm{~g}, 0.669 \mathrm{mmol})$ and tin(II) dichloride dihydrate $(0.756 \mathrm{~g}, 3.35 \mathrm{mmol})$ in methanol $(9.0 \mathrm{~mL})$. Purification by flash column chromatography eluting with $0-20 \%$ ethyl acetate in dichloromethane gave methyl (2S)-2-[(benzyloxycarbonyl)amino]-3[(5'-methoxy-2'-aminophenyl)amino]propanoate $(\mathbf{1 6 b})$ as a brown solid $(0.149 \mathrm{~g}, 60 \%)$. The next step was carried out as described previously using methyl (2S)-2-(benzyloxycarbonyl)amino]-3-[(5'methoxy-2'-aminophenyl)amino]propanoate $(\mathbf{1 6 b}) \quad(0.147 \mathrm{~g}, 0.394$ $\mathrm{mmol})$, $p$-toluenesulfonic acid $(0.224 \mathrm{~g}, 1.18 \mathrm{mmol})$ and polymersupported nitrite $\left(0.338 \mathrm{~g}\right.$, containing $1.18 \mathrm{mmol}$ of $\left.\mathrm{NO}_{2}^{-}\right)$in acetonitrile $(6.0 \mathrm{~mL})$. Purification by flash column chromatography eluting with $0-20 \%$ ethyl acetate in dichloromethane gave methyl (2S)-2-[(benzyloxycarbonyl)amino]-3-(6'-methoxy-1H-benzo[d][1.2.3] triazol-1'-yl)propanoate $(\mathbf{1 7 b})$ as a brown oil $(0.056 \mathrm{~g}, 39 \%)$. IR (neat) $3333,2953,1717,1622,1505,1233,1020,698 \mathrm{~cm}^{-1}$; $[\alpha]_{\mathrm{D}}^{23}$ $+17.7\left(c 1.0, \mathrm{CHCl}_{3}\right) ;{ }^{1} \mathrm{H}$ NMR $\left(400 \mathrm{MHz}, \mathrm{CDCl}_{3}\right): \delta 3.73(\mathrm{~s}, 3 \mathrm{H})$, $3.80(\mathrm{~s}, 3 \mathrm{H}), 4.88(\mathrm{dt}, J=7.6,4.4 \mathrm{~Hz}, 1 \mathrm{H}), 4.99(\mathrm{dd}, J=14.5,4.4 \mathrm{~Hz}$, $1 \mathrm{H}), 5.08(\mathrm{dd}, J=14.5,4.4 \mathrm{~Hz}, 1 \mathrm{H}), 5.10(\mathrm{~s}, 2 \mathrm{H}), 5.79(\mathrm{~d}, J=7.6 \mathrm{~Hz}$, $1 \mathrm{H}), 6.80(\mathrm{~d}, J=2.2 \mathrm{~Hz}, 1 \mathrm{H}), 6.98(\mathrm{dd}, J=9.0,2.2 \mathrm{~Hz}, 1 \mathrm{H}), 7.22-$ $7.40(\mathrm{~m}, 5 \mathrm{H}), 7.85(\mathrm{~d}, J=9.0 \mathrm{~Hz}, 1 \mathrm{H}) ;{ }^{13} \mathrm{C}\left\{{ }^{1} \mathrm{H}\right\} \mathrm{NMR}(101 \mathrm{MHz}$, $\left.\mathrm{CDCl}_{3}\right): \delta 48.4\left(\mathrm{CH}_{2}\right), 53.2\left(\mathrm{CH}_{3}\right), 54.1(\mathrm{CH}), 55.7\left(\mathrm{CH}_{3}\right), 67.2$ $\left(\mathrm{CH}_{2}\right), 89.4(\mathrm{CH}), 116.6(\mathrm{CH}), 120.7(\mathrm{CH}), 128.0(2 \times \mathrm{CH}), 128.3$ (CH), $128.6(2 \times \mathrm{CH}), 134.9(\mathrm{C}), 135.8(\mathrm{C}), 141.0(\mathrm{C}), 155.7(\mathrm{C})$, 160.3 (C), 169.5 (C); MS (ESI) $m / z: 407\left(\mathrm{M}+\mathrm{Na}^{+}, 100\right)$; HRMS (ESI): calcd for $\mathrm{C}_{19} \mathrm{H}_{20} \mathrm{~N}_{4} \mathrm{NaO}_{5}\left(\mathrm{M}+\mathrm{Na}^{+}\right)$, 407.1326; found, 407.1324.

Methyl (2S)-2-[(Benzyloxycarbonyl)amino]-3-(5'-bromo- $1 \mathrm{H}$ benzo[d][1.2.3]triazol-1'-yl)propanoate (17c). Methyl (2S)-2[(benzyloxycarbonyl)amino]-3-(5'-bromo-1H-benzo[d][1.2.3]triazol$1^{\prime}$-yl)propanoate $(17 \mathrm{c})$ was synthesized as described for methyl $(2 S)$ 2-[(benzyloxycarbonyl)amino]-3-(1H-benzo[d][1.2.3]triazol-1'-yl)propanoate (17a) using methyl (2S)-2-(benzyloxycarbonyl)amino]-3[(4'-bromo-2'-nitrophenyl)amino]propanoate $(\mathbf{1 5 c}) \quad(9.00 \mathrm{~g}, \quad 19.9$ $\mathrm{mmol})$ and tin(II) dichloride dihydrate $(22.0 \mathrm{~g}, 99.5 \mathrm{mmol})$ in methanol $(100 \mathrm{~mL})$. Purification by flash column chromatography eluting with $0-20 \%$ ethyl acetate in dichloromethane gave methyl (2S)-2-(benzyloxycarbonyl)amino]-3-[(4'-bromo-2' -aminophenyl)amino]propanoate (16c) as a brown oil $(6.42 \mathrm{~g}, 76 \%)$. The next step was carried out as described previously using methyl (2S)-2(benzyloxycarbonyl)amino]-3-[(4'-bromo-2'-aminophenyl)amino]propanoate $(16 \mathrm{c})(6.30 \mathrm{~g}, 14.9 \mathrm{mmol}), p$-toluenesulfonic acid $(8.50 \mathrm{~g}$, $44.7 \mathrm{mmol})$ and polymer-supported nitrite $(12.8 \mathrm{~g}$, containing 44.7 mmol of $\left.\mathrm{NO}_{2}^{-}\right)$in acetonitrile $(200 \mathrm{~mL})$. Purification by flash column chromatography eluting with $0-20 \%$ ethyl acetate in dichloromethane gave methyl (2S)-2-[(benzyloxycarbonyl)amino]-3-(5'bromo-1H-benzo[ $d][1.2 .3]$ triazol-1' $\left.1^{\prime}-y l\right)$ propanoate $(\mathbf{1 7 c})$ as a white solid (4.25 g, 66\%). mp 110-114 ${ }^{\circ} \mathrm{C}$; IR (neat) 3321, 2954, 1717, $1512,1211,1057,752 \mathrm{~cm}^{-1} ;[\alpha]_{\mathrm{D}}^{22}-22.2\left(\right.$ c $\left.1.0, \mathrm{CHCl}_{3}\right) ;{ }^{1} \mathrm{H}$ NMR 
$\left(500 \mathrm{MHz}, \mathrm{CDCl}_{3}\right): \delta 3.77(\mathrm{~s}, 3 \mathrm{H}), 4.84(\mathrm{dt}, J=6.6,4.5 \mathrm{~Hz}, 1 \mathrm{H})$, $5.00-5.20(\mathrm{~m}, 4 \mathrm{H}), 5.69(\mathrm{~d}, J=6.6 \mathrm{~Hz}, 1 \mathrm{H}), 7.22-7.47(\mathrm{~m}, 7 \mathrm{H})$, $8.15(\mathrm{~d}, J=1.0 \mathrm{~Hz}, 1 \mathrm{H}) ;{ }^{13} \mathrm{C}\left\{{ }^{1} \mathrm{H}\right\}$ NMR $\left(126 \mathrm{MHz}, \mathrm{CDCl}_{3}\right): \delta 48.6$ $\left(\mathrm{CH}_{2}\right), 53.3\left(\mathrm{CH}_{3}\right), 54.2(\mathrm{CH}), 67.3\left(\mathrm{CH}_{2}\right), 110.4(\mathrm{CH}), 117.4(\mathrm{C})$, $122.6(\mathrm{CH}), 128.3(2 \times \mathrm{CH}), 128.4(\mathrm{CH}), 128.6(2 \times \mathrm{CH}), 131.2$ (CH), 132.8 (C), 135.8 (C), 146.7 (C), 155.6 (C), 169.1 (C); MS (ESI) $m / z: 455\left(\mathrm{M}+\mathrm{Na}^{+}, 100\right)$; HRMS (ESI): calcd for $\mathrm{C}_{18} \mathrm{H}_{17}{ }^{79} \mathrm{BrN}_{4} \mathrm{NaO}_{4}\left(\mathrm{M}+\mathrm{Na}^{+}\right)$, 455.0325; found, 455.0325.

Methyl (2S)-2-[(Benzyloxycarbonyl)amino]-3-[5'-(trifluoromethyl)-1H-benzo[d][1.2.3]triazol-1'-yl]propanoate (17d). Methyl (2S)-2-[(benzyloxycarbonyl)amino]-3-[5'-(trifluoromethyl)$1 H$-benzo $[d][1.2 .3]$ triazol-1' $1^{\prime}$-yl] propanoate $(17 d)$ was synthesized as described for methyl (2S)-2-[(benzyloxycarbonyl)amino]-3-(1Hbenzo[d][1.2.3] triazol-1' ${ }^{\prime}$-yl)propanoate (17a) using methyl (2S)-2(benzyloxycarbonyl)amino]-3-\{[2'-nitro- $4^{\prime}$-(trifluoromethyl)phenyl]amino propanoate (15d) $(0.290 \mathrm{~g}, 0.657 \mathrm{mmol})$ and tin(II) dichloride dihydrate $(0.742 \mathrm{~g}, 3.29 \mathrm{mmol})$ in methanol $(9.0 \mathrm{~mL})$. Purification by flash column chromatography eluting with $0-20 \%$ ethyl acetate in dichloromethane gave methyl (2S)-2[(benzyloxycarbonyl)amino]-3-\{[2'-amino-4' -(trifluoromethyl)phenyl]amino propanoate $(\mathbf{1 6 d})$ as a white solid $(0.229 \mathrm{~g}, 85 \%)$. The next step was carried out as described previously using methyl (2S)-2(benzyloxycarbonyl)amino $]-3-\left\{\left[2^{\prime}\right.\right.$-amino- $4^{\prime}$ - (trifluoromethyl)phenyl]amino\}propanoate $(16 \mathrm{~d})(0.229 \mathrm{~g}, 5.57 \mathrm{mmol}), p$-toluenesulfonic acid $(0.313 \mathrm{~g}, 1.67 \mathrm{mmol})$ and polymer-supported nitrite $\left(0.477 \mathrm{~g}\right.$, containing $1.67 \mathrm{mmol}$ of $\left.\mathrm{NO}_{2}^{-}\right)$in acetonitrile $(9.0 \mathrm{~mL})$. Purification by flash column chromatography eluting with $20-40 \%$ ethyl acetate in petroleum ether (40-60) gave methyl (2S)-2[(benzyloxycarbonyl)amino]-3-[5'-(trifluoromethyl)-1H-benzo[d][1.2.3] triazol-1'-yl] propanoate $(\mathbf{1 7 d})$ as a white solid $(0.129 \mathrm{~g}, 57 \%)$. mp 130-132; IR (neat) 3333, 2957, 1742, 1717, 1526, 1331, 1209, $1123 \mathrm{~cm}^{-1} ;[\alpha]_{\mathrm{D}}^{23}+12.7$ (c 1.0, $\left.\mathrm{CHCl}_{3}\right) ;{ }^{1} \mathrm{H}$ NMR $(500 \mathrm{MHz}$, $\left.\mathrm{CDCl}_{3}\right): \delta 3.79(\mathrm{~s}, 3 \mathrm{H}), 4.85(\mathrm{dt}, J=6.4,4.4 \mathrm{~Hz}, 1 \mathrm{H}), 5.05(\mathrm{~d}, J=4.4$ $\mathrm{Hz}, 1 \mathrm{H}), 5.16(\mathrm{~d}, J=4.4 \mathrm{~Hz}, 1 \mathrm{H}), 5.18(\mathrm{~s}, 2 \mathrm{H}), 5.65(\mathrm{~d}, J=6.4 \mathrm{~Hz}$, $1 \mathrm{H}), 7.27-7.43(\mathrm{~m}, 5 \mathrm{H}), 7.48(\mathrm{~d}, J=8.7 \mathrm{~Hz}, 1 \mathrm{H}), 7.52(\mathrm{~d}, J=8.7$ $\mathrm{Hz}, 1 \mathrm{H}), 8.33(\mathrm{~s}, 1 \mathrm{H}) ;{ }^{13} \mathrm{C}\left\{{ }^{1} \mathrm{H}\right\} \mathrm{NMR}\left(126 \mathrm{MHz}, \mathrm{CDCl}_{3}\right): \delta 48.6$ $\left(\mathrm{CH}_{2}\right), 53.4\left(\mathrm{CH}_{3}\right), 54.3(\mathrm{CH}), 67.4\left(\mathrm{CH}_{2}\right), 110.2(\mathrm{CH}), 118.4(\mathrm{CH}$, $\left.\mathrm{q},{ }^{3} J_{\mathrm{CF}}=4.4 \mathrm{~Hz}\right), 124.0\left(\mathrm{C}, \mathrm{q},{ }^{1} J_{\mathrm{CF}}=272 \mathrm{~Hz}\right), 124.5\left(\mathrm{CH}, \mathrm{q},{ }^{3} J_{\mathrm{CF}}=\right.$ $3.0 \mathrm{~Hz}), 126.8\left(\mathrm{C}, \mathrm{q},{ }^{2} J_{\mathrm{CF}}=32.9 \mathrm{~Hz}\right), 128.4(2 \times \mathrm{CH}), 128.5(\mathrm{CH})$, $128.6(2 \times \mathrm{CH}), 135.3(\mathrm{C}), 135.8$ (C), 144.8 (C), 155.6 (C), 169.1 (C); MS (ESI) $m / z: 445\left(\mathrm{M}+\mathrm{Na}^{+}, 100\right)$; HRMS (ESI): calcd for $\mathrm{C}_{19} \mathrm{H}_{17} \mathrm{~F}_{3} \mathrm{~N}_{4} \mathrm{NaO}_{4}\left(\mathrm{M}+\mathrm{Na}^{+}\right)$, 445.1094; found, 445.1095.

Methyl (2S)-2-[(Benzyloxycarbonyl)amino]-3-(5', $6^{\prime}$-dichloro-1H-benzo[d][1.2.3]triazol-1'-yl)propanoate (17e). Methyl (2S)-2-[(benzyloxycarbonyl)amino]-3-(5',6'-dichloro- $1 H$ benzo[ $d][1.2 .3]$ triazol-1' ${ }^{\prime}$-yl)propanoate $(17 \mathbf{e})$ was synthesized as described for methyl (2S)-2-[(benzyloxycarbonyl)amino]-3-(1Hbenzo[d][1.2.3] triazol-1'-yl)propanoate (17a) using methyl (2S)-2(benzyloxycarbonyl)amino]-3-[(4',5'-dichloro-2' -nitrophenyl)amino]propanoate (15e) $(0.440 \mathrm{~g}, 0.669 \mathrm{mmol})$ and tin(II) dichloride dihydrate $(1.10 \mathrm{~g}, 4.98 \mathrm{mmol})$ in methanol $(14 \mathrm{~mL})$. Purification by flash column chromatography eluting with $0-20 \%$ ethyl acetate in dichloromethane gave methyl (2S)-2[(benzyloxycarbonyl)amino]-3-[(4',5'-dichloro-2'-aminophenyl)amino]propanoate (16e) as a brown oil $(0.365 \mathrm{~g}, 89 \%)$. The next step was carried out as described previously using methyl (2S)-2[(benzyloxycarbonyl $)$ amino $]-3-\left[\left(4^{\prime}, 5^{\prime}\right.\right.$-dichloro- $2^{\prime}$-aminophenyl $)$ amino]propanoate (16e) (0.365 g, $0.889 \mathrm{mmol}), p$-toluenesulfonic acid $(0.508 \mathrm{~g}, 2.67 \mathrm{mmol})$, and polymer-supported nitrite $(0.762 \mathrm{~g}$, containing $2.67 \mathrm{mmol}$ of $\left.\mathrm{NO}_{2}{ }^{-}\right)$in acetonitrile $(14 \mathrm{~mL})$. Purification by flash column chromatography eluting with $0-20 \%$ ethyl acetate in dichloromethane gave methyl (2S)-2-[(benzyloxycarbonyl)amino]-3$\left(5^{\prime}, 6^{\prime}\right.$-dichloro-1H-benzo[d][1.2.3] triazol-1'-yl)propanoate $(\mathbf{1 7 e})$ as a white solid $(0.304 \mathrm{~g}, 82 \%)$. mp $98-100{ }^{\circ} \mathrm{C}$; IR (neat) 3322,2957 , 1709, 1514, 1434, 1208, $751 \mathrm{~cm}^{-1}$; $[\alpha]_{\mathrm{D}}^{23}+11.2\left(c 1.0, \mathrm{CHCl}_{3}\right) ;{ }^{1} \mathrm{H}$ NMR $\left(400 \mathrm{MHz}, \mathrm{CDCl}_{3}\right): \delta 3.76(\mathrm{~s}, 3 \mathrm{H}), 4.84(\mathrm{dt}, J=7.0,4.5 \mathrm{~Hz}$, $1 \mathrm{H}), 4.97-5.15(\mathrm{~m}, 4 \mathrm{H}), 6.00-6.13(\mathrm{~m}, 1 \mathrm{H}), 7.21-7.36(\mathrm{~m}, 5 \mathrm{H})$, $7.67(\mathrm{~s}, 1 \mathrm{H}), 8.01(\mathrm{~s}, 1 \mathrm{H}) ;{ }^{13} \mathrm{C}\left\{{ }^{1} \mathrm{H}\right\}$ NMR $\left(101 \mathrm{MHz}, \mathrm{CDCl}_{3}\right): \delta 48.8$ $\left(\mathrm{CH}_{2}\right), 53.3\left(\mathrm{CH}_{3}\right), 54.2(\mathrm{CH}), 67.5\left(\mathrm{CH}_{2}\right), 110.7(\mathrm{CH}), 120.8$ $(\mathrm{CH}), 128.0(2 \times \mathrm{CH}), 128.3(\mathrm{CH}), 128.6(2 \times \mathrm{CH}), 128.9(\mathrm{C})$,
$132.9(2 \times \mathrm{C}), 135.7$ (C), 144.3 (C), 155.8 (C), 169.2 (C); MS (ESI) $m / z: 445\left(\mathrm{M}+\mathrm{Na}^{+}, 100\right)$; HRMS (ESI): calcd for $\mathrm{C}_{18} \mathrm{H}_{16}{ }^{35} \mathrm{Cl}_{2} \mathrm{~N}_{4} \mathrm{NaO}_{4}\left(\mathrm{M}+\mathrm{Na}^{+}\right), 445.0441$; found, 445.0432 .

Methyl (2S)-2-[(Benzyloxycarbonyl)amino]-3-(6'-chloro-5' methyl-1H-benzo[d][1.2.3]triazol-1'-yl)propanoate (17f). Methyl (2S)-2-[(benzyloxycarbonyl)amino]-3-(6'-chloro-5'-methyl$1 H$-benzo $[d][1.2 .3]$ triazol-1'-yl)propanoate $(\mathbf{1 7 f})$ was synthesized as described for methyl (2S)-2-[(benzyloxycarbonyl)amino]-3-(1Hbenzo[ $d][1.2 .3]$ triazol-1'-yl)propanoate (17a) using methyl (2S)-2(benzyloxycarbonyl)amino]-3-[(5'-chloro-4'-methyl-2'-nitrophenyl)amino]propanoate $(\mathbf{1 5 f})(0.520 \mathrm{~g}, 1.23 \mathrm{mmol})$ and tin(II) dichloride dihydrate $(1.40 \mathrm{~g}, 6.15 \mathrm{mmol})$ in methanol $(17 \mathrm{~mL})$. Purification by flash column chromatography, eluting with $0-20 \%$ ethyl acetate in dichloromethane, gave methyl (2S)-2-[(benzyloxycarbonyl)amino]-3[( $5^{\prime}$-chloro-4'-methyl-2'-aminophenyl)amino]propanoate $(\mathbf{1 6 f})$ as a brown oil $(0.315 \mathrm{~g}, 65 \%)$. The next step was carried out as described previously using methyl (2S)-2-(benzyloxycarbonyl)amino $]-3-\left[\left(5^{\prime}\right.\right.$ chloro-4'-methyl-2'-aminophenyl)amino]propanoate (16f) (0.315 g, $0.803 \mathrm{mmol}), p$-toluenesulfonic acid $(0.458 \mathrm{~g}, 2.41 \mathrm{mmol})$, and polymer-supported nitrite $\left(0.689 \mathrm{~g}\right.$, containing $2.41 \mathrm{mmol}$ of $\left.\mathrm{NO}_{2}^{-}\right)$ in acetonitrile $(13 \mathrm{~mL})$. Purification by flash column chromatography eluting with $0-20 \%$ ethyl acetate in dichloromethane gave methyl (2S)-2-[(benzyloxycarbonyl) amino]-3- ( $6^{\prime}$-chloro-5' -methyl- $1 \mathrm{H}$ benzo[d][1.2.3] triazol-1'-yl)propanoate $(\mathbf{1 7 f})$ as a white solid $(0.247$ g, $78 \%$ ). mp $86-88^{\circ} \mathrm{C}$; IR (neat) $3310,2955,1747,1713,1516$, 1437, 1213, $754 \mathrm{~cm}^{-1} ;[\alpha]_{\mathrm{D}}^{23}+13.7\left(c 1.0, \mathrm{CHCl}_{3}\right) ;{ }^{1} \mathrm{H}$ NMR $(500$ $\left.\mathrm{MHz}, \mathrm{CDCl}_{3}\right): \delta 2.40(\mathrm{~s}, 3 \mathrm{H}), 3.74(\mathrm{~s}, 3 \mathrm{H}), 4.84(\mathrm{dt}, J=7.4,4.8 \mathrm{~Hz}$, $1 \mathrm{H}), 5.03(\mathrm{br} \mathrm{d}, J=4.8 \mathrm{~Hz}, 2 \mathrm{H}), 5.08(\mathrm{~s}, 2 \mathrm{H}), 6.08-6.23(\mathrm{~m}, 1 \mathrm{H})$, 7.18-7.38 (m, 5H), $7.52(\mathrm{~s}, 1 \mathrm{H}), 7.70(\mathrm{~s}, 1 \mathrm{H}) ;{ }^{13} \mathrm{C}\left\{{ }^{1} \mathrm{H}\right\}$ NMR $(101$ $\left.\mathrm{MHz}, \mathrm{CDCl}_{3}\right): \delta 20.7\left(\mathrm{CH}_{3}\right), 48.5\left(\mathrm{CH}_{2}\right), 53.1\left(\mathrm{CH}_{3}\right), 54.2(\mathrm{CH})$, $67.3\left(\mathrm{CH}_{2}\right), 109.2(\mathrm{CH}), 120.3(\mathrm{CH}), 128.1(2 \times \mathrm{CH}), 128.3(\mathrm{CH})$, $128.5(2 \times \mathrm{CH}), 132.5(\mathrm{C}), 132.9$ (C), $135.3(\mathrm{C}), 135.8(\mathrm{C}), 144.4$ (C), 155.8 (C), 169.4 (C); MS (ESI) $m / z: 425\left(\mathrm{M}+\mathrm{Na}^{+}, 100\right)$; HRMS (ESI): calcd for $\mathrm{C}_{19} \mathrm{H}_{19}{ }^{35} \mathrm{ClN}_{4} \mathrm{NaO}_{4}\left(\mathrm{M}+\mathrm{Na}^{+}\right)$, 425.0987; found, 425.0975 .

(2S)-2-Amino-3-(1H-benzo[d][1.2.3]triazol-1'-yl)propanoic Acid Hydrochloride (18a). A solution of methyl (2S)-2[(benzyloxycarbonyl)amino]-3-(1H-benzo[d][1.2.3]triazol-1'-yl)propanoate $(\mathbf{1 7 a})(0.0830 \mathrm{~g}, 0.236 \mathrm{mmol})$ in $6 \mathrm{M}$ aqueous hydrochloric acid solution $(10 \mathrm{~mL})$ was heated under reflux for 20 h. After cooling to ambient temperature, the reaction mixture was concentrated in vacuo, and the resulting residue recrystallized from methanol and diethyl ether to afford (2S)-2-amino-3-(1H-benzo[d][1.2.3] triazol-1'-yl)propanoic acid hydrochloride (18a) as a pale brown solid (0.054 g, 94\%). mp 190-192 ${ }^{\circ} \mathrm{C}$; IR (neat) 2893, 2739, $1728,1234,1165,756 \mathrm{~cm}^{-1} ;[\alpha]_{\mathrm{D}}^{24}+11.9(c 0.7, \mathrm{MeOH}) ;{ }^{1} \mathrm{H}$ NMR $\left(400 \mathrm{MHz}, \mathrm{CD}_{3} \mathrm{OD}\right): \delta 4.79(\mathrm{dd}, J=5.8,4.1 \mathrm{~Hz}, 1 \mathrm{H}), 5.26(\mathrm{dd}, J=$ $15.5,4.1 \mathrm{~Hz}, 1 \mathrm{H}), 5.37$ (dd, $J=15.5,5.8 \mathrm{~Hz}, 1 \mathrm{H}), 7.49$ (ddd, $J=8.4$, 7.0, $0.9 \mathrm{~Hz}, 1 \mathrm{H}), 7.63(\mathrm{ddd}, J=8.5,7.0,0.9 \mathrm{~Hz}, 1 \mathrm{H}), 7.84(\mathrm{dt}, J=8.5$, $0.9 \mathrm{~Hz}, 1 \mathrm{H}), 8.05(\mathrm{dt}, J=8.4,0.9 \mathrm{~Hz}, 1 \mathrm{H}) ;{ }^{13} \mathrm{C}\left\{{ }^{1} \mathrm{H}\right\}$ NMR $(101$ $\left.\mathrm{MHz}, \mathrm{CD}_{3} \mathrm{OD}\right): \delta 48.0\left(\mathrm{CH}_{2}\right), 53.5(\mathrm{CH}), 111.1(\mathrm{CH}), 120.3(\mathrm{CH})$, $126.0(\mathrm{CH}), 129.5(\mathrm{CH}), 134.9$ (C), 146.8 (C), 168.9 (C); MS (ESI) $m / z: 229\left(\mathrm{M}+\mathrm{Na}^{+}, 100\right)$; HRMS (ESI): calcd for $\mathrm{C}_{9} \mathrm{H}_{10} \mathrm{~N}_{4} \mathrm{NaO}_{2}(\mathrm{M}$ $\left.+\mathrm{Na}^{+}\right)$, 229.0696; found, 229.0706.

(2S)-2-Amino-3-(6'-methoxy-1 H-benzo[d][1.2.3]triazol-1'yl)propanoic Acid Hydrochloride (18b). (2S)-2-Amino-3-(6'methoxy-1H-benzo[d][1.2.3] triazol-1'-yl)propanoic acid hydrochloride (18b) was synthesized as described for (2S)-2-amino-3-(1Hbenzo[d][1.2.3] triazol-1'-yl)propanoic acid hydrochloride (18a) using methyl (2S)-2-[(benzyloxycarbonyl)amino]-3-(6'-methoxy$1 H$-benzo[d][1.2.3] triazol-1'-yl)propanoate (17b) (0.056 g, 0.146 $\mathrm{mmol}$ ) in $6 \mathrm{M}$ aqueous hydrochloric acid solution $(11 \mathrm{~mL})$. Purification by recrystallization from methanol and diethyl ether gave (2S)-2-amino-3-(6'-methoxy-1H-benzo[ $d][1.2 .3]$ triazol-1' ${ }^{\prime}$-yl)propanoic acid hydrochloride $(\mathbf{1 8 b})$ as a pale brown solid $(0.023 \mathrm{~g}$, $67 \%$ ). $\mathrm{mp} 179-180{ }^{\circ} \mathrm{C}$ (decomposition); IR (neat) $3403,2895,1746$, 1624, 1506, 1236, $820 \mathrm{~cm}^{-1}$; $[\alpha]_{\mathrm{D}}^{24}+7.3(\mathrm{c} 1.0, \mathrm{MeOH}) ;{ }^{1} \mathrm{H}$ NMR $\left(400 \mathrm{MHz}, \mathrm{CD}_{3} \mathrm{OD}\right): \delta 4.77(\mathrm{dd}, J=5.4,4.2 \mathrm{~Hz}, 1 \mathrm{H}), 5.21(\mathrm{dd}, J=$ $15.4,4.2 \mathrm{~Hz}, 1 \mathrm{H}), 5.31(\mathrm{dd}, J=15.4,5.4 \mathrm{~Hz}, 1 \mathrm{H}), 7.08(\mathrm{dd}, J=9.1$, $2.2 \mathrm{~Hz}, 1 \mathrm{H}), 7.25(\mathrm{~d}, J=2.2 \mathrm{~Hz}, 1 \mathrm{H}), 7.86(\mathrm{~d}, J=9.1 \mathrm{~Hz}, 1 \mathrm{H})$; 
${ }^{13} \mathrm{C}\left\{{ }^{1} \mathrm{H}\right\}$ NMR (101 MHz, CD $\left.{ }_{3} \mathrm{OD}\right): \delta 46.4\left(\mathrm{CH}_{2}\right), 52.2(\mathrm{CH}), 55.2$ $\left(\mathrm{CH}_{3}\right), 89.9(\mathrm{CH}), 117.3(\mathrm{CH}), 119.5(\mathrm{CH}), 134.9(\mathrm{C}), 140.6(\mathrm{C})$, 160.9 (C), 167.7 (C); MS (ESI) $m / z: 259\left(\mathrm{M}+\mathrm{Na}^{+}, 100\right)$; HRMS (ESI): calcd for $\mathrm{C}_{10} \mathrm{H}_{12} \mathrm{~N}_{4} \mathrm{NaO}_{3}\left(\mathrm{M}+\mathrm{Na}^{+}\right), 259.0802$; found, 259.0808 .

(2S)-2-Amino-3-(5'-bromo-1H-benzo[d][1.2.3]triazol-1' -yl)propanoic Acid Hydrochloride (18c). (2S)-2-Amino-3-(5'-bromo$1 H$-benzo[d][1.2.3] triazol-1'-yl)propanoic acid hydrochloride $(18 \mathrm{c})$ was synthesized as described for (2S)-2-amino-3-(1H-benzo[d][1.2.3] triazol-1'-yl)propanoic acid hydrochloride (18a) using methyl (2S)-2-[(benzyloxycarbonyl)amino]-3-(5'-bromo- $1 H$-benzo $[d]$ [1.2.3] triazol-1'-yl)propanoate $(17 \mathrm{c})(0.200 \mathrm{~g}, 0.462 \mathrm{mmol})$ in $6 \mathrm{M}$ aqueous hydrochloric acid solution $(20 \mathrm{~mL})$. Purification by recrystallization from methanol and diethyl ether gave (2S)-2amino-3-(5'-bromo- $1 H$-benzo[d][1.2.3] triazol-1'-yl)propanoic acid hydrochloride (18c) as a pale brown solid $(0.103 \mathrm{~g}, 78 \%) . \mathrm{mp}$ 168-172 ${ }^{\circ} \mathrm{C}$; IR (neat) 3387, 2847, 1744, 1474, 1211, $802 \mathrm{~cm}^{-1}$; $[\alpha]_{\mathrm{D}}^{22}+9.1(c 0.7, \mathrm{MeOH}) ;{ }^{1} \mathrm{H}$ NMR $\left(500 \mathrm{MHz}, \mathrm{CD}_{3} \mathrm{OD}\right): \delta 4.74$ $(\mathrm{dd}, J=5.9,4.0 \mathrm{~Hz}, 1 \mathrm{H}), 5.24(\mathrm{dd}, J=15.5,4.0 \mathrm{~Hz}, 1 \mathrm{H}), 5.34(\mathrm{dd}, J$ $=15.5,5.9 \mathrm{~Hz}, 1 \mathrm{H}), 7.73(\mathrm{dd}, J=8.8,1.7 \mathrm{~Hz}, 1 \mathrm{H}), 7.78(\mathrm{dd}, J=8.8$, $0.5 \mathrm{~Hz}, 1 \mathrm{H}), 8.26(\mathrm{dd}, J=1.7,0.5 \mathrm{~Hz}, 1 \mathrm{H}) ;{ }^{13} \mathrm{C}\left\{{ }^{1} \mathrm{H}\right\}$ NMR $(126$ $\left.\mathrm{MHz}, \mathrm{CD}_{3} \mathrm{OD}\right): \delta 48.3\left(\mathrm{CH}_{2}\right), 53.6(\mathrm{CH}), 112.9(\mathrm{CH}), 119.0(\mathrm{C})$, $123.1(\mathrm{CH}), 132.7(\mathrm{CH}), 134.0(\mathrm{C}), 148.1(\mathrm{C}), 169.0(\mathrm{C})$; MS (ESI) $m / z: 285\left(\mathrm{M}+\mathrm{H}^{+}, 100\right)$; HRMS (ESI): calcd for $\mathrm{C}_{9} \mathrm{H}_{10}{ }^{79} \mathrm{BrN}_{4} \mathrm{O}_{2}(\mathrm{M}$ $\left.+\mathrm{H}^{+}\right)$, 284.9982; found, 284.9978 .

(2S)-2-Amino-3-[5'-(trifluoromethyl)- $1 H$-benzo[d][1.2.3]triazol-1'-yl]propanoic Acid Hydrochloride (18d). (2S)-2Amino-3-[5'-(trifluoromethyl)-1H-benzo[d][1.2.3] triazol-1' ${ }^{\prime}$-yl]propanoic acid hydrochloride (18d) was synthesized as described for (2S)-2-amino-3-(1H-benzo[d][1.2.3] triazol-1'-yl)propanoic acid hydrochloride (18a) using methyl (2S)-2-[(benzyloxycarbonyl)amino]3-[5'-(trifluoromethyl)-1H-benzo[d][1.2.3] triazol-1'-yl]propanoate (17d) $(0.120 \mathrm{~g}, 0.284 \mathrm{mmol})$ in $6 \mathrm{M}$ aqueous hydrochloric acid solution $(20 \mathrm{~mL})$. Purification by recrystallization from methanol and diethyl ether gave (2S)-2-amino-3-[ $5^{\prime}$-(trifluoromethyl)-1H-benzo[d][1.2.3]triazol-1'-yl]propanoic acid hydrochloride $(\mathbf{1 8 d})$ as a pale brown solid (0.046 g, 59\%). mp $185-187{ }^{\circ} \mathrm{C}$ (decomposition); IR (neat) $3381,2916,1743,1331,1215,1125,816 \mathrm{~cm}^{-1} ;[\alpha]_{\mathrm{D}}^{24}+12.0(c$ 1.0, $\mathrm{MeOH}) ;{ }^{1} \mathrm{H}$ NMR $\left(400 \mathrm{MHz}, \mathrm{CD}_{3} \mathrm{OD}\right): \delta 4.83$ (br t, $J=4.7 \mathrm{~Hz}$, $1 \mathrm{H}), 5.36(\mathrm{dd}, J=15.5,4.7 \mathrm{~Hz}, 1 \mathrm{H}), 5.45(\mathrm{dd}, J=15.5,4.7 \mathrm{~Hz}, 1 \mathrm{H})$, $7.88(\mathrm{dd}, J=8.8,0.9 \mathrm{~Hz}, 1 \mathrm{H}), 8.09(\mathrm{~d}, J=8.8 \mathrm{~Hz}, 1 \mathrm{H}), 8.42(\mathrm{br} \mathrm{s}$, $1 \mathrm{H}) ;{ }^{13} \mathrm{C}\left\{{ }^{1} \mathrm{H}\right\}$ NMR $\left(101 \mathrm{MHz}, \mathrm{CD}_{3} \mathrm{OD}\right): \delta 47.0\left(\mathrm{CH}_{2}\right), 52.2(\mathrm{CH})$, $111.6(\mathrm{CH}), 117.3\left(\mathrm{CH}, \mathrm{q},{ }^{3} J_{\mathrm{CF}}=4.5 \mathrm{~Hz}\right), 124.2\left(\mathrm{C}, \mathrm{q},{ }^{1} J_{\mathrm{CF}}=271\right.$ $\mathrm{Hz}), 124.3\left(\mathrm{CH}, \mathrm{q},{ }^{3} J_{\mathrm{CF}}=3.2 \mathrm{~Hz}\right), 126.8\left(\mathrm{C}, \mathrm{q},{ }^{2} J_{\mathrm{CF}}=32.7 \mathrm{~Hz}\right), 135.2$ (C), 144.7 (C), 167.6 (C); MS (ESI) $m / z: 297\left(\mathrm{M}+\mathrm{Na}^{+}, 100\right)$; HRMS (ESI): calcd for $\mathrm{C}_{10} \mathrm{H}_{9} \mathrm{~F}_{3} \mathrm{~N}_{4} \mathrm{NaO}_{2}\left(\mathrm{M}+\mathrm{Na}^{+}\right)$, 297.0570; found, 297.0574.

(2S)-2-Amino-3-(5',6'-dichloro-1H-benzo[d][1.2.3]triazol-1' yl)propanoic Acid Hydrochloride (18e). (2S)-2-Amino-3-(5', $6^{\prime}$ dichloro-1H-benzo[d][1.2.3] triazol-1'-yl)propanoic acid hydrochloride (18e) was synthesized as described for (2S)-2-amino-3-(1Hbenzo[d][1.2.3] triazol-1'-yl)propanoic acid hydrochloride (18a) using methyl (2S)-2-[(benzyloxycarbonyl)amino]-3- $\left(5^{\prime}, 6^{\prime}\right.$-dichloro$1 H$-benzo[d][1.2.3] triazol-1'-yl)propanoate (17e) (0.290 g, 0.685 $\mathrm{mmol}$ ) in $6 \mathrm{M}$ aqueous hydrochloric acid solution $(48 \mathrm{~mL})$. Purification by recrystallization from methanol and diethyl ether gave (2S)-2-amino-3-( $5^{\prime}, 6^{\prime}$-dichloro- $1 H$-benzo[ $\left.d\right][1.2 .3]$ triazol-1' yl)propanoic acid hydrochloride (18e) as a pale brown solid (0.167 g, $88 \%$ ). mp $180-182{ }^{\circ} \mathrm{C}$ (decomposition); IR (neat) 3381, 2875, $1740,1507,1434,1214,822 \mathrm{~cm}^{-1} ;[\alpha]_{\mathrm{D}}^{23}+11.4($ c 1.0, MeOH$) ;{ }^{1} \mathrm{H}$ NMR (400 MHz, CD 30 OD): $\delta 4.81(\mathrm{br} \mathrm{t}, J=4.8 \mathrm{~Hz}, 1 \mathrm{H}), 5.29(\mathrm{dd}, J$ $=15.5,4.8 \mathrm{~Hz}, 1 \mathrm{H}), 5.38(\mathrm{dd}, J=15.5,4.8 \mathrm{~Hz}, 1 \mathrm{H}), 8.18(\mathrm{~s}, 1 \mathrm{H})$, $8.24(\mathrm{~s}, 1 \mathrm{H}) ;{ }^{13} \mathrm{C}\left\{{ }^{1} \mathrm{H}\right\}$ NMR (101 MHz, $\left.\mathrm{CD}_{3} \mathrm{OD}\right): \delta 47.0\left(\mathrm{CH}_{2}\right)$, $52.1(\mathrm{CH}), 111.8(\mathrm{CH}), 120.1(\mathrm{CH}), 128.9(\mathrm{C}), 132.6(\mathrm{C}), 132.7$ (C), 144.4 (C), 167.5 (C); MS (ESI) $m / z: 297\left(\mathrm{M}+\mathrm{Na}^{+}, 100\right)$; HRMS (ESI): calcd for $\mathrm{C}_{9} \mathrm{H}_{8}{ }^{35} \mathrm{Cl}_{2} \mathrm{~N}_{4} \mathrm{NaO}_{2}\left(\mathrm{M}+\mathrm{Na}^{+}\right)$, 296.9917; found, 296.9915.

(2S)-2-Amino-3-(6'-chloro-5' -methyl-1H-benzo[d][1.2.3]triazol-1'-yl)propanoic Acid Hydrochloride (18f). (2S)-2Amino-3-(6'-chloro-5' -methyl-1H-benzo[d][1.2.3]triazol-1'-yl)- propanoic acid hydrochloride (18f) was synthesized as described for (2S)-2-amino-3-(1H-benzo[d][1.2.3] triazol-1'-yl)propanoic acid hydrochloride (18a) using methyl (2S)-2-[(benzyloxycarbonyl)amino]3-(6'-chloro-5' -methyl-1H-benzo[d][1.2.3] triazol-1'-yl)propanoate (17f) $(0.240 \mathrm{~g}, 0.596 \mathrm{mmol})$ in $6 \mathrm{M}$ aqueous hydrochloric acid solution $(42 \mathrm{~mL})$. Purification by recrystallization from methanol and diethyl ether gave (2S)-2-amino-3-(6'-chloro-5'-methyl-1H-benzo[d][1.2.3] triazol-1'-yl)propanoic acid hydrochloride $(\mathbf{1 8 f})$ as a pale brown solid (0.139 g, 92\%). mp 199-201 ${ }^{\circ} \mathrm{C}$ (decomposition); IR (neat) 3376, 2951, 1744, 1454, 1228, 1001, $865 \mathrm{~cm}^{-1}$; $[\alpha]_{\mathrm{D}}^{23}+11.1(c$ 1.0, $\mathrm{MeOH}$ ); ${ }^{1} \mathrm{H}$ NMR (400 MHz, $\left.\mathrm{CD}_{3} \mathrm{OD}\right): \delta 2.53(\mathrm{~s}, 3 \mathrm{H}), 4.78$ $(\mathrm{dd}, J=5.5,4.2 \mathrm{~Hz}, 1 \mathrm{H}), 5.22(\mathrm{dd}, J=15.5,4.2 \mathrm{~Hz}, 1 \mathrm{H}), 5.32(\mathrm{dd}, J$ $=15.5,5.5 \mathrm{~Hz}, 1 \mathrm{H}), 7.94(\mathrm{~s}, 1 \mathrm{H}), 7.95(\mathrm{~s}, 1 \mathrm{H}) ;{ }^{13} \mathrm{C}\left\{{ }^{1} \mathrm{H}\right\}$ NMR $(101$ $\left.\mathrm{MHz}, \mathrm{CD}_{3} \mathrm{OD}\right): \delta 19.4\left(\mathrm{CH}_{3}\right), 46.7\left(\mathrm{CH}_{2}\right), 52.1(\mathrm{CH}), 110.0(\mathrm{CH})$, $119.6(\mathrm{CH}), 132.7$ (C), 133.3 (C), 135.5 (C), 144.5 (C), 167.5 (C); MS (ESI) $m / z: 255\left(\mathrm{M}+\mathrm{H}^{+}, 100\right)$; HRMS (ESI): calcd for $\mathrm{C}_{10} \mathrm{H}_{12}{ }^{35} \mathrm{ClN}_{4} \mathrm{O}_{2}\left(\mathrm{M}+\mathrm{H}^{+}\right)$, 255.0643; found, 255.0651 .

Methyl (2S)-2-[(Benzyloxycarbonyl)amino]-3-[5'-(4" -methoxyphenyl)-1 $\mathrm{H}$-benzo[d][1.2.3] triazol-1'-yl]propanoate (19a). To a vial containing 4-methoxyphenylboronic acid (0.105 g, $0.693 \mathrm{mmol})$, potassium fluoride $(0.0805 \mathrm{~g}, 1.39 \mathrm{mmol})$, and $\left[1,1^{\prime}\right.$ bis(diphenylphosphino)ferrocene]palladium(II) dichloride-dichloromethane complex $(0.0289 \mathrm{~g}, 0.0346 \mathrm{mmol})$ were added a solution of methyl (2S)-2-[(benzyloxycarbonyl)amino]-3-(5'-bromo- $1 H$-benzo$[d][1.2 .3]$ triazol-1'-yl)propanoate $(17 \mathrm{c})(0.200 \mathrm{~g}, 0.462 \mathrm{mmol})$ in 1,4-dioxane $(3.6 \mathrm{~mL})$ and water $(0.4 \mathrm{~mL})$. The reaction mixture was degassed with argon for $0.15 \mathrm{~h}$, and the vial was sealed under argon and stirred at $80{ }^{\circ} \mathrm{C}$ for $20 \mathrm{~h}$. The reaction mixture was filtered through Celite and washed with ethyl acetate $(50 \mathrm{~mL})$. The filtrate was washed with $1 \mathrm{M}$ aqueous hydrochloric acid $(50 \mathrm{~mL})$, water $(50$ $\mathrm{mL})$, and brine $(50 \mathrm{~mL})$, dried $\left(\mathrm{MgSO}_{4}\right)$, and concentrated in vacuo to give the crude product. Purification with flash chromatography using silica gel eluting with $15 \%$ diethyl ether in dichloromethane gave methyl (2S)-2-[(benzyloxycarbonyl)amino]-3-[5'-(4"-methoxyphenyl)-1H-benzo[d][1.2.3] triazol-1'-yl]propanoate $(19 a)$ as a white solid (0.179 g, 84\%). mp 139-142 ${ }^{\circ} \mathrm{C}$; IR (neat) 3333, 2932, 1721, 1605, $1512,1342,1250,1026 \mathrm{~cm}^{-1} ;[\alpha]_{\mathrm{D}}^{21}+7.6\left(\mathrm{c} 1.0, \mathrm{CHCl}_{3}\right) ;{ }^{1} \mathrm{H}$ NMR $\left(400 \mathrm{MHz}, \mathrm{CDCl}_{3}\right): \delta 3.77(\mathrm{~s}, 3 \mathrm{H}), 3.87(\mathrm{~s}, 3 \mathrm{H}), 4.89(\mathrm{dt}, J=7.2,4.3$ $\mathrm{Hz}, 1 \mathrm{H}), 5.04-5.22(\mathrm{~m}, 4 \mathrm{H}), 5.68(\mathrm{~d}, J=7.2 \mathrm{~Hz}, 1 \mathrm{H}), 7.02(\mathrm{~d}, J=$ $8.8 \mathrm{~Hz}, 2 \mathrm{H}), 7.28-7.40(\mathrm{~m}, 5 \mathrm{H}), 7.43(\mathrm{br} \mathrm{d}, J=8.7 \mathrm{~Hz}, 1 \mathrm{H}), 7.55(\mathrm{~d}$, $J=8.8 \mathrm{~Hz}, 2 \mathrm{H}), 7.59(\mathrm{dd}, J=8.7,1.5 \mathrm{~Hz}, 1 \mathrm{H}), 8.13(\mathrm{dd}, J=1.5,0.7$ $\mathrm{Hz}, 1 \mathrm{H}) ;{ }^{13} \mathrm{C}\left\{{ }^{1} \mathrm{H}\right\}$ NMR $\left(101 \mathrm{MHz}, \mathrm{CDCl}_{3}\right): \delta 48.6\left(\mathrm{CH}_{2}\right), 53.2$ $\left(\mathrm{CH}_{3}\right), 54.3(\mathrm{CH}), 55.4\left(\mathrm{CH}_{3}\right), 67.3\left(\mathrm{CH}_{2}\right), 109.2(\mathrm{CH}), 114.4(2 \times$ $\mathrm{CH}), 117.2(\mathrm{CH}), 127.9(\mathrm{CH}), 128.3(2 \times \mathrm{CH}), 128.4(\mathrm{CH}), 128.5$ $(2 \times \mathrm{CH}), 128.6(2 \times \mathrm{CH}), 132.9(2 \times \mathrm{C}), 135.9(\mathrm{C}), 137.6(\mathrm{C})$, 146.5 (C), 155.7 (C), 159.4 (C), 169.3 (C); MS (ESI) $m / z: 483$ (M $\left.+\mathrm{Na}^{+}, 100\right)$; HRMS (ESI): calcd for $\mathrm{C}_{25} \mathrm{H}_{24} \mathrm{~N}_{4} \mathrm{NaO}_{5}\left(\mathrm{M}+\mathrm{Na}^{+}\right)$, 483.1639; found, 483.1623.

Methyl (2S)-2-[(Benzyloxycarbonyl)amino]-3-[5'-(3",4"methylenedioxyphenyl)-1H-benzo[d][1.2.3]triazol-1'-yl]propanoate (19b). Methyl (2S)-2-[(benzyloxycarbonyl)amino]-3[5'-( $3^{\prime \prime}, 4^{\prime \prime}$-methylenedioxyphenyl)- $1 H$-benzo[d] [1.2.3] triazol-1' -yl]propanoate (19b) was synthesized as described for methyl (2S)-2[(benzyloxycarbonyl)amino]-3-[5'-(4" -methoxyphenyl)- $1 H$-benzo[d][1.2.3] triazol-1'-yl]propanoate (19a) using methyl (2S)-2[(benzyloxycarbonyl)amino]-3-(5'-bromo- $1 H$-benzo[d][1.2.3]triazol$1^{\prime}$-yl)propanoate $(17 \mathrm{c})(0.104 \mathrm{~g}, 0.239 \mathrm{mmol}), 3,4-$ (methylenedioxy)phenylboronic acid $(0.0793 \mathrm{~g}, 0.478 \mathrm{mmol}),\left[1,1^{\prime}-\right.$ bis(diphenylphosphino)ferrocene]palladium(II) dichloride-dichloromethane complex $(0.0146 \mathrm{~g}, 0.0179 \mathrm{mmol})$, and potassium fluoride $(0.0417 \mathrm{~g}, 0.717 \mathrm{mmol})$ in dioxane $(3.6 \mathrm{~mL})$ and water $(0.4 \mathrm{~mL})$. Purification with flash chromatography using silica gel eluting with $5 \%$ diethyl ether in dichloromethane gave methyl (2S)-2[(benzyloxycarbonyl)amino]-3-[ $5^{\prime}-\left(3^{\prime \prime}, 4^{\prime \prime}\right.$-methylenedioxyphenyl)$1 H$-benzo[d][1.2.3] triazol-1'-yl]propanoate $(19 \mathbf{b})$ as a white solid $(0.0926 \mathrm{~g}, 82 \%)$. mp $160-162{ }^{\circ} \mathrm{C}$; IR (neat) $3322,2930,1711,1510$, $1480,1225,1038,799,735 \mathrm{~cm}^{-1} ;[\alpha]_{\mathrm{D}}^{24}+13.4\left(c 1.0, \mathrm{CHCl}_{3}\right) ;{ }^{1} \mathrm{H}$ NMR $\left(400 \mathrm{MHz}, \mathrm{CDCl}_{3}\right): \delta 3.78(\mathrm{~s}, 3 \mathrm{H}), 4.89(\mathrm{dt}, J=7.0,4.3 \mathrm{~Hz}$, $1 \mathrm{H}), 5.03-5.22(\mathrm{~m}, 4 \mathrm{H}), 5.65(\mathrm{~d}, J=7.0 \mathrm{~Hz}, 1 \mathrm{H}), 6.03(\mathrm{~s}, 2 \mathrm{H}), 6.92$ (dd, $J=7.5,0.9 \mathrm{~Hz}, 1 \mathrm{H}), 7.04-7.11(\mathrm{~m}, 2 \mathrm{H}), 7.29-7.40(\mathrm{~m}, 5 \mathrm{H})$, 
$7.42(\mathrm{br} \mathrm{d}, J=8.6 \mathrm{~Hz}, 1 \mathrm{H}), 7.54(\mathrm{dd}, J=8.6,1.3 \mathrm{~Hz}, 1 \mathrm{H}), 8.10(\mathrm{~d}, J=$ $1.3 \mathrm{~Hz}, 1 \mathrm{H}) ;{ }^{13} \mathrm{C}\left\{{ }^{1} \mathrm{H}\right\} \mathrm{NMR}\left(101 \mathrm{MHz}, \mathrm{CDCl}_{3}\right): \delta 48.6\left(\mathrm{CH}_{2}\right), 53.3$ $\left(\mathrm{CH}_{3}\right), 54.3(\mathrm{CH}), 67.3\left(\mathrm{CH}_{2}\right), 101.3\left(\mathrm{CH}_{2}\right), 108.0(\mathrm{CH}), 108.7$ $(\mathrm{CH}), 109.2(\mathrm{CH}), 117.4(\mathrm{CH}), 121.1(\mathrm{CH}), 128.0(\mathrm{CH}), 128.3(2$ $\times \mathrm{CH}), 128.4(\mathrm{CH}), 128.6(2 \times \mathrm{CH}), 133.1(\mathrm{C}), 134.7(\mathrm{C}), 135.9$ (C), 137.7 (C), 146.4 (C), 147.4 (C), 148.3 (C), 155.7 (C), 169.3 (C); MS (ESI) $m / z: 497\left(\mathrm{M}+\mathrm{Na}^{+}, 100\right)$; HRMS (ESI): calcd for $\mathrm{C}_{25} \mathrm{H}_{22} \mathrm{~N}_{4} \mathrm{NaO}_{6}\left(\mathrm{M}+\mathrm{Na}^{+}\right)$, 497.1432; found, 497.1411.

Methyl (2S)-2-[(Benzyloxycarbonyl)amino]-3-[5'-(4"-morpholinophenyl)-1H-benzo[d][1.2.3] triazol-1'-yl]propanoate (19c). Methyl (2S)-2-[(benzyloxycarbonyl)amino]-3-[5'-(4" -morpholinophenyl)-1H-benzo[d][1.2.3] triazol-1'-yl]propanoate (19c) was synthesized as described for methyl (2S)-2-[(benzyloxycarbonyl)amino $]-3-\left[5^{\prime}-\left(4^{\prime \prime}-\right.\right.$ methoxyphenyl)-1H-benzo[ $\left.d\right][1.2 .3]$ triazol-1' ${ }^{\prime}$-yl $]$ propanoate (19a) using methyl (2S)-2-[(benzyloxycarbonyl)amino]3-(5'-bromo-1H-benzo[d][1.2.3] triazol-1'-yl)propanoate $(\mathbf{1 7 c})$ $(0.0810 \mathrm{~g}, 0.187 \mathrm{mmol})$, 4-morpholinophenylboronic acid $(0.116 \mathrm{~g}$, $0.561 \mathrm{mmol}),\left[1,1^{\prime}\right.$-bis(diphenylphosphino)ferrocene $]$ palladium(II) dichloride-dichloromethane complex $(0.0115 \mathrm{~g}, 0.0140 \mathrm{mmol})$, and potassium fluoride $(0.0326 \mathrm{~g}, 0.561 \mathrm{mmol})$ in dioxane $(3.6 \mathrm{~mL})$ and water $(0.4 \mathrm{~mL})$. Purification with flash chromatography using silica gel eluting with $20 \%$ diethyl ether in dichloromethane gave methyl (2S)-2-[(benzyloxycarbonyl)amino]-3-[ $5^{\prime}$ - ( $4^{\prime \prime}$-morpholinophenyl)$1 H$-benzo[d][1.2.3] triazol-1'-yl]propanoate $(19 \mathrm{c})$ as a white solid (0.0578 g, 60\%). mp 159-160 ${ }^{\circ} \mathrm{C}$; IR (neat) $3302,2953,1744,1717$, $1522,1485,1227,1117,926,729 \mathrm{~cm}^{-1} ;[\alpha]_{\mathrm{D}}^{20}+5.3\left(c 1.0, \mathrm{CHCl}_{3}\right)$; ${ }^{1} \mathrm{H}$ NMR $\left(400 \mathrm{MHz}, \mathrm{CDCl}_{3}\right): \delta 3.23(\mathrm{t}, J=4.5 \mathrm{~Hz}, 4 \mathrm{H}), 3.76(\mathrm{~s}$, $3 \mathrm{H}), 3.90(\mathrm{t}, J=4.5 \mathrm{~Hz}, 4 \mathrm{H}), 4.88(\mathrm{dt}, J=7.1,4.3 \mathrm{~Hz}, 1 \mathrm{H}), 5.04-$ $5.24(\mathrm{~m}, 4 \mathrm{H}), 5.76(\mathrm{~d}, J=7.1 \mathrm{~Hz}, 1 \mathrm{H}), 7.04(\mathrm{~d}, J=8.5 \mathrm{~Hz}, 2 \mathrm{H})$, $7.29-7.39(\mathrm{~m}, 5 \mathrm{H}), 7.43(\mathrm{~d}, J=8.7 \mathrm{~Hz}, 1 \mathrm{H}), 7.55(\mathrm{~d}, J=8.5 \mathrm{~Hz}$, $2 \mathrm{H}), 7.59(\mathrm{dd}, J=8.7,1.4 \mathrm{~Hz}, 1 \mathrm{H}), 8.13($ br s, $1 \mathrm{H}) ;{ }^{13} \mathrm{C}\left\{{ }^{1} \mathrm{H}\right\} \mathrm{NMR}$ $\left(101 \mathrm{MHz}, \mathrm{CDCl}_{3}\right): \delta 48.6\left(\mathrm{CH}_{2}\right), 49.3\left(2 \times \mathrm{CH}_{2}\right), 53.2\left(\mathrm{CH}_{3}\right), 54.3$ $(\mathrm{CH}), 66.7\left(2 \times \mathrm{CH}_{2}\right), 67.3\left(\mathrm{CH}_{2}\right), 109.2(\mathrm{CH}), 116.1(2 \times \mathrm{CH})$, $116.9(\mathrm{CH}), 127.7(\mathrm{CH}), 128.2(2 \times \mathrm{CH}), 128.3(3 \times \mathrm{CH}), 128.6(2$ $\times \mathrm{CH}), 132.0(\mathrm{C}), 132.9$ (C), 135.9 (C), 137.5 (C), 146.6 (C), 150.5 (C), 155.7 (C), 169.4 (C); MS (ESI) $m / z: 538\left(\mathrm{M}+\mathrm{Na}^{+}, 100\right)$; HRMS (ESI): calcd for $\mathrm{C}_{28} \mathrm{H}_{29} \mathrm{~N}_{5} \mathrm{NaO}_{5}\left(\mathrm{M}+\mathrm{Na}^{+}\right)$, 538.2061; found, 538.2039.

Methyl (2S)-2-[(Benzyloxycarbonyl)amino]-3-[5'-(4"-nitrophenyl)-1H-benzo[d][1.2.3]triazol-1'-yl]propanoate (19d). Methyl (2S)-2-[(benzyloxycarbonyl)amino]-3-[5'-(4" -nitrophenyl)$1 H$-benzo[d][1.2.3] triazol-1'-yl]propanoate $(19 \mathrm{~d})$ was synthesized as described for methyl (2S)-2-[(benzyloxycarbonyl)amino]-3-[5'(4" -methoxyphenyl)-1H-benzo[d][1.2.3] triazol-1'-yl]propanoate (19a) using methyl (2S)-2-[(benzyloxycarbonyl)amino]-3-(5' bromo-1H-benzo[d][1.2.3] triazol-1'-yl)propanoate $(17 \mathrm{c})(0.105 \mathrm{~g}$, $0.241 \mathrm{mmol})$, 4-nitrophenylboronic acid $(0.0806 \mathrm{~g}, 0.483 \mathrm{mmol})$, $\left[1,1^{\prime}\right.$-bis(diphenylphosphino)ferrocene $]$ palladium(II) dichloride-dichloromethane complex $(0.0148 \mathrm{~g}, 0.0181 \mathrm{mmol})$, and potassium fluoride $(0.0420 \mathrm{~g}, 0.723 \mathrm{mmol})$ in dioxane $(3.6 \mathrm{~mL})$ and water $(0.4$ $\mathrm{mL}$ ). Purification with flash chromatography using silica gel eluting with $5 \%$ diethyl ether in dichloromethane gave methyl (2S)-2[(benzyloxycarbonyl)amino]-3-[5'-(4" -nitrophenyl)-1H-benzo[d][1.2.3] triazol-1'-yl]propanoate $(19 \mathrm{~d})$ as a white solid $(0.0805 \mathrm{~g}$, $70 \%$ ). mp $178-180{ }^{\circ} \mathrm{C}$; IR (neat) $3480,2950,1721,1512,1342$, 1219, $748 \mathrm{~cm}^{-1} ;[\alpha]_{\mathrm{D}}^{20}-46.3\left(c 1.0, \mathrm{CHCl}_{3}\right) ;{ }^{1} \mathrm{H}$ NMR $(400 \mathrm{MHz}$, $\left.\mathrm{CDCl}_{3}\right): \delta 3.81(\mathrm{~s}, 3 \mathrm{H}), 4.90(\mathrm{dt}, J=6.8,4.2 \mathrm{~Hz}, 1 \mathrm{H}), 5.02-5.25(\mathrm{~m}$, $4 \mathrm{H}), 5.60(\mathrm{~d}, J=6.8 \mathrm{~Hz}, 1 \mathrm{H}), 7.30-7.42(\mathrm{~m}, 5 \mathrm{H}), 7.52(\mathrm{br} \mathrm{d}, J=8.7$ $\mathrm{Hz}, 1 \mathrm{H}), 7.60(\mathrm{dd} J=8.7,1.3 \mathrm{~Hz}, 1 \mathrm{H}), 7.77(\mathrm{~d}, J=8.9 \mathrm{~Hz}, 2 \mathrm{H}), 8.27$ $(\mathrm{dd}, J=1.3,0.8 \mathrm{~Hz}, 1 \mathrm{H}), 8.35(\mathrm{~d}, J=8.9 \mathrm{~Hz}, 2 \mathrm{H}) ;{ }^{13} \mathrm{C}\left\{{ }^{1} \mathrm{H}\right\} \mathrm{NMR}$ $\left(101 \mathrm{MHz}, \mathrm{CDCl}_{3}\right): \delta 48.7\left(\mathrm{CH}_{2}\right), 53.4\left(\mathrm{CH}_{3}\right), 54.3(\mathrm{CH}), 67.3$ $\left(\mathrm{CH}_{2}\right), 110.0(\mathrm{CH}), 118.9(\mathrm{CH}), 124.3(2 \times \mathrm{CH}), 127.7(\mathrm{CH})$, $128.2(2 \times \mathrm{CH}), 128.3(2 \times \mathrm{CH}), 128.4(\mathrm{CH}), 128.6(2 \times \mathrm{CH})$, 134.0 (C), 135.4 (C), 135.9 (C), 146.3 (C), 146.8 (C), 147.3 (C), 155.6 (C), 169.2 (C); MS (ESI) $m / z: 498\left(\mathrm{M}+\mathrm{Na}^{+}, 100\right)$; HRMS (ESI): calcd for $\mathrm{C}_{24} \mathrm{H}_{21} \mathrm{~N}_{5} \mathrm{NaO}_{6}\left(\mathrm{M}+\mathrm{Na}^{+}\right), 498.1384$; found, 498.1365.

Methyl (2S)-2-[(Benzyloxycarbonyl)amino]-3-[5'-(naphthalen-2"-yl)-1H-benzo[d][1.2.3]triazol-1'-yl]propanoate (19e). Methyl (2S)-2-[(benzyloxycarbonyl)amino]-3-[5' -(naphthalen-2" -
yl)-1H-benzo $[d][1.2 .3]$ triazol-1'-yl]propanoate $(19 e)$ was synthesized as described for methyl (2S)-2-[(benzyloxycarbonyl)amino]-3[ $5^{\prime}$-(4"-methoxyphenyl)-1H-benzo[d][1.2.3] triazol-1'-yl]propanoate (19a) using methyl (2S)-2-[(benzyloxycarbonyl)amino]-3-(5'bromo-1H-benzo[ $d][1.2 .3]$ triazol-1' ${ }^{\prime}$-yl $)$ propanoate $(\mathbf{1 7 c})(0.105 \mathrm{~g}$, $0.242 \mathrm{mmol}), 2$-naphthylboronic acid $(0.0834 \mathrm{~g}, 0.485 \mathrm{mmol})$, [1, $1^{\prime}$ bis(diphenylphosphino)ferrocene]palladium(II) dichloride-dichloromethane complex $(0.0148 \mathrm{~g}, 0.0182 \mathrm{mmol})$, and potassium fluoride $(0.0422 \mathrm{~g}, 0.726 \mathrm{mmol})$ in dioxane $(3.6 \mathrm{~mL})$ and water $(0.4 \mathrm{~mL})$. Purification with flash chromatography using silica gel eluting with $5 \%$ diethyl ether in dichloromethane gave methyl (2S)-2[(benzyloxycarbonyl)amino]-3-[ $5^{\prime}$-(naphthalen-2"-yl)-1H-benzo[d][1.2.3] triazol-1'-yl]propanoate $(19 \mathrm{e})$ as a white solid $(0.105 \mathrm{~g}, 91 \%)$. $\mathrm{mp} 72-74{ }^{\circ} \mathrm{C}$; IR (neat) $3335,2950,1713,1508,1211,1018,777$, $729 \mathrm{~cm}^{-1} ;[\alpha]_{\mathrm{D}}^{20}+11.7\left(c 1.0, \mathrm{CHCl}_{3}\right) ;{ }^{1} \mathrm{H} \mathrm{NMR}\left(400 \mathrm{MHz}, \mathrm{CDCl}_{3}\right)$ : $\delta 3.81(\mathrm{~s}, 3 \mathrm{H}), 4.93(\mathrm{dt}, J=7.1,4.3 \mathrm{~Hz}, 1 \mathrm{H}), 5.02-5.27(\mathrm{~m}, 4 \mathrm{H})$, $5.76(\mathrm{~d}, J=7.1 \mathrm{~Hz}, 1 \mathrm{H}), 7.25-7.62(\mathrm{~m}, 11 \mathrm{H}), 7.81(\mathrm{~d}, J=8.4 \mathrm{~Hz}$, $1 \mathrm{H}), 7.90$ (br d, $J=8.4 \mathrm{~Hz}, 1 \mathrm{H}), 7.93(\mathrm{~d}, J=8.1 \mathrm{~Hz}, 1 \mathrm{H}), 8.13$ (br s, $1 \mathrm{H}) ;{ }^{13} \mathrm{C}\left\{{ }^{1} \mathrm{H}\right\}$ NMR $\left(101 \mathrm{MHz}, \mathrm{CDCl}_{3}\right): \delta 48.7\left(\mathrm{CH}_{2}\right), 53.3\left(\mathrm{CH}_{3}\right)$, $54.3(\mathrm{CH}), 67.3\left(\mathrm{CH}_{2}\right), 108.7(\mathrm{CH}), 120.8(\mathrm{CH}), 125.4(\mathrm{CH}), 125.7$ $(\mathrm{CH}), 126.0(\mathrm{CH}), 126.3(\mathrm{CH}), 127.5(\mathrm{CH}), 128.1(\mathrm{CH}), 128.2(2$ $\times \mathrm{CH}), 128.4(2 \times \mathrm{CH}), 128.6(2 \times \mathrm{CH}), 130.7(\mathrm{CH}), 131.7(\mathrm{C})$, 133.2 (C), 133.8 (C), 135.9 (C), 137.1 (C), 139.1 (C), 146.0 (C), 155.7 (C), 169.4 (C); MS (ESI) $m / z: 503\left(\mathrm{M}+\mathrm{Na}^{+}, 100\right)$; HRMS (ESI): calcd for $\mathrm{C}_{28} \mathrm{H}_{24} \mathrm{~N}_{4} \mathrm{NaO}_{4}\left(\mathrm{M}+\mathrm{Na}^{+}\right)$, 503.1690; found, 503.1671

Methyl (2S)-2-[(Benzyloxycarbonyl)amino]-3-[5'-(thiophen3"-yl)-1H-benzo[d][1.2.3]triazol-1'-yl]propanoate (19f). Methyl (2S)-2-[(benzyloxycarbonyl)amino]-3-[5' -(thiophen-3" -yl)-1Hbenzo[d][1.2.3] triazol-1'-yl]propanoate (19f) was synthesized as described for methyl (2S)-2-[(benzyloxycarbonyl)amino]-3-[5'-(4"methoxyphenyl)-1H-benzo[d][1.2.3] triazol-1'-yl]propanoate (19a) using methyl (2S)-2-[(benzyloxycarbonyl)amino]-3-(5'-bromo- $1 H$ benzo $[d][1.2 .3]$ triazol-1'-yl)propanoate $(17 \mathrm{c})(0.111 \mathrm{~g}, 0.256$ $\mathrm{mmol})$, 3-thiopheneboronic acid $(0.0654 \mathrm{~g}, 0.511 \mathrm{mmol}),\left[1,1^{\prime}\right.$ bis(diphenylphosphino)ferrocene]palladium(II) dichloride-dichloromethane complex $(0.0157 \mathrm{~g}, 0.0192 \mathrm{mmol})$, and potassium fluoride $(0.0446 \mathrm{~g}, 0.768 \mathrm{mmol})$ in dioxane $(3.6 \mathrm{~mL})$ and water $(0.4 \mathrm{~mL})$. Purification with flash chromatography using silica gel eluting with $5 \%$ diethyl ether in dichloromethane gave methyl (2S)-2[(benzyloxycarbonyl)amino]-3-[ $5^{\prime}$-(thiophen-3" -yl)-1H-benzo[d][1.2.3] triazol-1'-yl]propanoate (19f) as a white solid $(0.0826 \mathrm{~g}, 74 \%)$. mp $144-145^{\circ} \mathrm{C}$; IR (neat) $3321,2957,1744,1713,1514,1214,788$ $\mathrm{cm}^{-1} ;[\alpha]_{\mathrm{D}}^{23}+18.0\left(\mathrm{c} 1.0, \mathrm{CHCl}_{3}\right) ;{ }^{1} \mathrm{H} \mathrm{NMR}\left(400 \mathrm{MHz}, \mathrm{CDCl}_{3}\right): \delta$ $3.77(\mathrm{~s}, 3 \mathrm{H}), 4.89(\mathrm{dt}, J=7.1,4.3 \mathrm{~Hz}, 1 \mathrm{H}), 5.02-5.25(\mathrm{~m}, 4 \mathrm{H}), 5.64$ $(\mathrm{d}, J=7.1 \mathrm{~Hz}, 1 \mathrm{H}), 7.28-7.47(\mathrm{~m}, 8 \mathrm{H}), 7.49(\mathrm{dd}, J=2.8,1.5 \mathrm{~Hz}$, $1 \mathrm{H}), 7.62(\mathrm{dd}, J=8.7,1.3 \mathrm{~Hz}, 1 \mathrm{H}), 8.20(\mathrm{dd}, J=1.3,0.7 \mathrm{~Hz}, 1 \mathrm{H})$; ${ }^{13} \mathrm{C}\left\{{ }^{1} \mathrm{H}\right\}$ NMR $\left(101 \mathrm{MHz}, \mathrm{CDCl}_{3}\right): \delta 48.6\left(\mathrm{CH}_{2}\right), 53.3\left(\mathrm{CH}_{3}\right), 54.3$ $(\mathrm{CH}), 67.3\left(\mathrm{CH}_{2}\right), 109.4(\mathrm{CH}), 116.9(\mathrm{CH}), 120.9(\mathrm{CH}), 126.5$ $(\mathrm{CH}), 126.7(\mathrm{CH}), 127.5(\mathrm{CH}), 128.3\left(2 \times \mathrm{CH}_{2}\right), 128.4(\mathrm{CH})$, $128.6\left(2 \times \mathrm{CH}_{2}\right), 132.5(\mathrm{C}), 133.1(\mathrm{C}), 135.9(\mathrm{C}), 141.5(\mathrm{C}), 146.4$ (C), 155.7 (C), 169.3 (C); MS (ESI) $m / z: 459\left(\mathrm{M}+\mathrm{Na}^{+}, 100\right)$; HRMS (ESI): calcd for $\mathrm{C}_{22} \mathrm{H}_{20} \mathrm{~N}_{4} \mathrm{NaO}_{4} \mathrm{~S}\left(\mathrm{M}+\mathrm{Na}^{+}\right)$, 459.1097; found, 459.1081 .

(2S)-2-Amino-3-[5'-(4"-methoxyphenyl)-1H-benzo[d][1.2.3]triazol-1'-yl]propanoic Acid Hydrochloride (21a). To a solution of methyl (2S)-2-[(benzyloxycarbonyl)amino]-3-[5'-(4" methoxyphenyl)-1H-benzo[d][1.2.3] triazol-1' -yl] propanoate (19a) $(0.155 \mathrm{~g}, 0.336 \mathrm{mmol})$ in a mixture of methanol $(12 \mathrm{~mL})$ and $1,4-$ dioxane $(6 \mathrm{~mL})$ was added a solution of cesium carbonate $(0.142 \mathrm{~g}$, $0.436 \mathrm{mmol})$ in water $(6 \mathrm{~mL})$. The reaction mixture was stirred at room temperature for $20 \mathrm{~h}$ and then concentrated in vacuo. The resulting residue was dissolved in water $(100 \mathrm{~mL})$ and acidified to $\mathrm{pH}$ 1 with $1 \mathrm{M}$ aqueous hydrochloric acid. The aqueous layer was extracted with dichloromethane $(3 \times 30 \mathrm{~mL})$, and the combined organic layers were washed with $1 \mathrm{M}$ aqueous hydrochloric acid (50 $\mathrm{mL})$, dried $\left(\mathrm{MgSO}_{4}\right)$, and concentrated in vacuo to give (2S)-2[(benzyloxycarbonyl)amino]-3-[ $5^{\prime}-\left(4^{\prime \prime}\right.$-methoxyphenyl)- $1 H$-benzo[d][1.2.3] triazol-1'-yl]propanoic acid (20a) as a white solid. This was used for the next reaction without any further purification. The 
resulting solid was dissolved in 1,4-dioxane $(1 \mathrm{~mL})$, and $6 \mathrm{M}$ aqueous hydrochloric acid $(5 \mathrm{~mL})$ was added. The reaction mixture was heated under reflux for $1 \mathrm{~h}$ and then concentrated in vacuo to give the crude product. Purification by recrystallization from methanol and diethyl ether gave (2S)-2-amino-3-[5'-(4" -methoxyphenyl)- $1 H$ benzo $[d][1.2 .3]$ triazol-1' ${ }^{\prime}$-yl $]$ propanoic acid hydrochloride (21a) as a white solid $(0.0906 \mathrm{~g}, 77 \%)$. mp $214-216{ }^{\circ} \mathrm{C}$; IR (neat) 2832, 1744, 1612, 1489, 1250, $810 \mathrm{~cm}^{-1} ;[\alpha]_{\mathrm{D}}^{20}+6.5(c 0.5, \mathrm{MeOH}) ;{ }^{1} \mathrm{H}$ NMR ( $\left.400 \mathrm{MHz}, \mathrm{CD}_{3} \mathrm{OD}\right): \delta 3.85(\mathrm{~s}, 3 \mathrm{H}), 4.79(\mathrm{dd}, J=5.7,4.1 \mathrm{~Hz}$, $1 \mathrm{H}), 5.27(\mathrm{dd}, J=15.5,4.1 \mathrm{~Hz}, 1 \mathrm{H}), 5.37(\mathrm{dd}, J=15.5,5.7 \mathrm{~Hz}, 1 \mathrm{H})$, $7.04(\mathrm{~d}, J=8.8 \mathrm{~Hz}, 2 \mathrm{H}), 7.63(\mathrm{~d}, J=8.8 \mathrm{~Hz}, 2 \mathrm{H}), 7.81-7.92(\mathrm{~m}$, $2 \mathrm{H}), 8.15$ (br s, $1 \mathrm{H}) ;{ }^{13} \mathrm{C}\left\{{ }^{1} \mathrm{H}\right\}$ NMR $\left(101 \mathrm{MHz}, \mathrm{CD}_{3} \mathrm{OD}\right): \delta 46.8$ $\left(\mathrm{CH}_{2}\right), 52.2(\mathrm{CH}), 54.4\left(\mathrm{CH}_{3}\right), 110.0(\mathrm{CH}), 114.1(2 \times \mathrm{CH}), 115.6$ $(\mathrm{CH}), 128.0(\mathrm{CH}), 128.1(2 \times \mathrm{CH}), 132.4(\mathrm{C}), 132.6(\mathrm{C}), 138.4$ (C), 146.3 (C), 159.8 (C), 167.6 (C); MS (ESI) $m / z: 313\left(\mathrm{M}+\mathrm{H}^{+}\right.$, 100); HRMS (ESI): calcd for $\mathrm{C}_{16} \mathrm{H}_{17} \mathrm{~N}_{4} \mathrm{O}_{3}\left(\mathrm{M}+\mathrm{H}^{+}\right)$, 313.1295; found, 313.1290 .

(2S)-2-Amino-3-[5'-(3", 4"-methylenedioxyphenyl)-1Hbenzo[d][1.2.3]triazol-1'-yl]propanoic Acid Hydrochloride (21b). (2S)-2-Amino-3-[5'-(3",4"-methylenedioxyphenyl)-1H-benzo[d][1.2.3] triazol-1'-yl]propanoic acid hydrochloride (21b) was synthesized as described for (2S)-2-amino-3-[5'-(4"-methoxyphenyl)-1H-benzo[d][1.2.3] triazol-1'-yl]propanoic acid hydrochloride (21a) using methyl (2S)-2-[(benzyloxycarbonyl)amino]-3-[5'$\left(3^{\prime \prime}, 4^{\prime \prime}\right.$-methylenedioxyphenyl)-1H-benzo[ $\left.d\right][1.2 .3]$ triazol-1' ${ }^{\prime}$-yl $]$ propanoate $(19 b)(0.0898 \mathrm{~g}, 0.189 \mathrm{mmol})$ and cesium carbonate $(0.0801 \mathrm{~g}, 0.246 \mathrm{mmol})$. Purification by recrystallization from methanol and diethyl ether gave $(2 S)-2$-amino-3-[5'-( $\left(3^{\prime \prime}, 4^{\prime \prime}\right.$-methylenedioxyphenyl)- $1 H$-benzo[d][1.2.3] triazol-1'-yl]propanoic acid hydrochloride $(\mathbf{2 1 b})$ as a white solid $(0.0610 \mathrm{~g}, 89 \%)$. mp 203-205 ${ }^{\circ} \mathrm{C}$; IR (neat) 2886, 1724, 1479, 1248, 1227, 1038, $800 \mathrm{~cm}^{-1}$; $[\alpha]_{\mathrm{D}}^{20}+17.4$ (c $0.5, \mathrm{MeOH}) ;{ }^{1} \mathrm{H}$ NMR $\left(400 \mathrm{MHz}, \mathrm{CD}_{3} \mathrm{OD}\right): \delta 4.80$ (dd, $J=5.7$, $4.1 \mathrm{~Hz}, 1 \mathrm{H}), 5.27(\mathrm{dd}, J=15.5,4.1 \mathrm{~Hz}, 1 \mathrm{H}), 5.37(\mathrm{dd}, J=15.5,5.7$ $\mathrm{Hz}, 1 \mathrm{H}), 6.01(\mathrm{~s}, 2 \mathrm{H}), 6.94(\mathrm{~d}, J=7.8,0.5 \mathrm{~Hz}, 1 \mathrm{H}), 7.14-7.26(\mathrm{~m}$, $2 \mathrm{H}), 7.81-7.95(\mathrm{~m}, 2 \mathrm{H}), 8.14$ (br s, $1 \mathrm{H}) ;{ }^{13} \mathrm{C}\left\{{ }^{1} \mathrm{H}\right\}$ NMR $(101 \mathrm{MHz}$, $\left.\mathrm{CD}_{3} \mathrm{OD}\right): \delta 46.7\left(\mathrm{CH}_{2}\right), 52.2(\mathrm{CH}), 101.3\left(\mathrm{CH}_{2}\right), 107.3(\mathrm{CH}), 108.3$ $(\mathrm{CH}), 109.9(\mathrm{CH}), 116.0(\mathrm{CH}), 120.8(\mathrm{CH}), 128.1(\mathrm{CH}), 132.7$ (C), 134.3 (C), 138.5 (C), 146.2 (C), 147.7 (C), 148.5 (C), 167.5 (C); MS (ESI) $m / z: 327\left(\mathrm{M}+\mathrm{H}^{+}, 100\right)$; HRMS (ESI): calcd for $\mathrm{C}_{16} \mathrm{H}_{15} \mathrm{~N}_{4} \mathrm{O}_{4}\left(\mathrm{M}+\mathrm{H}^{+}\right)$, 327.1088; found, 327.1074.

(2S)-2-Amino-3-[5'-(4" -morpholinophenyl)-1H-benzo[d][1.2.3]triazol-1'-yl]propanoic Acid Hydrochloride (21c). (2S)-2Amino-3-[5'-(4"-morpholinophenyl)-1H-benzo[d][1.2.3]triazol-1' yl]propanoic acid hydrochloride (21c) was synthesized as described for (2S)-2-amino-3-[ $5^{\prime}$-( $4^{\prime \prime}$-methoxyphenyl)-1H-benzo[d][1.2.3]triazol-1'-yl]propanoic acid hydrochloride (21a) using methyl $(2 S)$ 2 -[(benzyloxycarbonyl)amino]-3-[5'-(4"-morpholinophenyl)-1Hbenzo $[d][1.2 .3]$ triazol-1' ${ }^{\prime}$-yl $]$ propanoate $(19 \mathrm{c})(0.116 \mathrm{~g}, 0.224 \mathrm{mmol})$ and cesium carbonate $(0.0948 \mathrm{~g}, 0.291 \mathrm{mmol})$. Purification by recrystallization from methanol and diethyl ether gave (2S)-2-amino3 -[5'-(4" -morpholinophenyl)- $1 H$-benzo $[d][1.2 .3]$ triazol-1' -yl]propanoic acid hydrochloride $(\mathbf{2 1 c})$ as a white solid $(0.0957 \mathrm{~g}, 97 \%)$. mp $172-174{ }^{\circ} \mathrm{C}$; IR (neat) $3366,2866,1738,1487,1121,1059,889$, $806 \mathrm{~cm}^{-1} ;[\alpha]_{\mathrm{D}}^{20}+13.5($ c $1.0, \mathrm{MeOH}) ;{ }^{1} \mathrm{H}$ NMR $(400 \mathrm{MHz}$, $\left.\mathrm{CD}_{3} \mathrm{OD}\right): \delta 3.79(\mathrm{t}, J=4.6 \mathrm{~Hz}, 4 \mathrm{H}), 4.17(\mathrm{t}, J=4.6 \mathrm{~Hz}, 4 \mathrm{H}), 4.83$ $(\mathrm{dd}, J=5.6,4.2 \mathrm{~Hz}, 1 \mathrm{H}), 5.31(\mathrm{dd}, J=15.5,4.2 \mathrm{~Hz}, 1 \mathrm{H}), 5.41(\mathrm{dd}, J$ $=15.5,5.6,1 \mathrm{H}), 7.89(\mathrm{~d}, J=8.8 \mathrm{~Hz}, 2 \mathrm{H}), 7.93-8.01(\mathrm{~m}, 4 \mathrm{H}), 8.31$ (br s, $1 \mathrm{H}) ;{ }^{13} \mathrm{C}\left\{{ }^{1} \mathrm{H}\right\}$ NMR (101 MHz, $\left.\mathrm{CD}_{3} \mathrm{OD}\right): \delta 46.8\left(\mathrm{CH}_{2}\right), 52.1$ $(\mathrm{CH}), 54.9\left(2 \times \mathrm{CH}_{2}\right), 64.1\left(2 \times \mathrm{CH}_{2}\right), 110.6(\mathrm{CH}), 117.2(\mathrm{CH})$, $121.5(2 \times \mathrm{CH}), 127.9(\mathrm{CH}), 129.1(2 \times \mathrm{CH}), 133.4(\mathrm{C}), 136.3(\mathrm{C})$, 141.3 (C), 141.9 (C), 146.2 (C), 167.5 (C); MS (ESI) $m / z: 368$ (M $\left.+\mathrm{H}^{+}, 100\right)$; HRMS (ESI): calcd for $\mathrm{C}_{19} \mathrm{H}_{22} \mathrm{~N}_{5} \mathrm{O}_{3}\left(\mathrm{M}+\mathrm{H}^{+}\right)$, 368.1717; found, 368.1708.

(2S)-2-Amino-3-[5'-(4" -nitrophenyl)-1H-benzo[d][1.2.3]triazol-1'-yl]propanoic Acid Hydrochloride (21d). (2S)-2Amino-3-[5'-(4"'-nitrophenyl)-1H-benzo[d][1.2.3] triazol-1'-yl]propanoic acid hydrochloride (21d) was synthesized as described for (2S)-2-amino-3-[5'-(4" -methoxyphenyl)-1H-benzo[ $d][1.2 .3]$ triazol$1^{\prime}$-yl]propanoic acid hydrochloride (21a) using methyl (2S)-2[(benzyloxycarbonyl)amino]-3-[5'-(4"'-nitrophenyl)-1H-benzo[d]-
[1.2.3] triazol-1'-yl]propanoate $(19 \mathrm{~d})(0.0529 \mathrm{~g}, 0.111 \mathrm{mmol})$ and cesium carbonate $(0.0469 \mathrm{~g}, 0.144 \mathrm{mmol})$. Purification by recrystallization from methanol and diethyl ether gave (2S)-2amino-3-[ $5^{\prime}$-( $4^{\prime \prime}$-nitrophenyl)-1H-benzo[d][1.2.3] triazol-1'-yl]propanoic acid hydrochloride (21d) as a white solid $(0.0375 \mathrm{~g}, 93 \%)$. mp 168-170 ${ }^{\circ} \mathrm{C}$; IR (neat) 2899, 1735, 1595, 1514, 1344, $750 \mathrm{~cm}^{-1}$; $[\alpha]_{\mathrm{D}}^{20}+80.0(c 0.5, \mathrm{MeOH}) ;{ }^{1} \mathrm{H}$ NMR $\left(400 \mathrm{MHz}, \mathrm{CD}_{3} \mathrm{OD}\right): \delta 4.81$ $(\mathrm{dd}, J=5.7,4.1 \mathrm{~Hz}, 1 \mathrm{H}), 5.31(\mathrm{dd}, J=15.5,4.1 \mathrm{~Hz}, 1 \mathrm{H}), 5.41(\mathrm{dd}, J$ $=15.5,5.7 \mathrm{~Hz}, 1 \mathrm{H}), 7.94-8.06(\mathrm{~m}, 4 \mathrm{H}), 8.36(\mathrm{~d}, J=8.9 \mathrm{~Hz}, 2 \mathrm{H})$, 8.39 (br s, $1 \mathrm{H}) ;{ }^{13} \mathrm{C}\left\{{ }^{1} \mathrm{H}\right\}$ NMR $\left(101 \mathrm{MHz}, \mathrm{CD}_{3} \mathrm{OD}\right): \delta 46.8\left(\mathrm{CH}_{2}\right)$, $52.2(\mathrm{CH}), 110.7(\mathrm{CH}), 117.8(\mathrm{CH}), 123.8(2 \times \mathrm{CH}), 127.9(\mathrm{CH})$, $128.1(2 \times \mathrm{CH}), 133.7(\mathrm{C}), 136.0(\mathrm{C}), 146.2(\mathrm{C}), 146.4(\mathrm{C}), 147.4$ (C), 167.6 (C); MS (ESI) $m / z: 328\left(\mathrm{M}+\mathrm{H}^{+}, 100\right)$; HRMS (ESI): calcd for $\mathrm{C}_{15} \mathrm{H}_{14} \mathrm{~N}_{5} \mathrm{O}_{4}\left(\mathrm{M}+\mathrm{H}^{+}\right)$, 328.1040; found, 328.1035 .

(2S)-2-Amino-3-[5'-(naphthalen-2"'-yl)-1H-benzo[d][1.2.3]triazol-1'-yl]propanoic Acid Hydrochloride (21e). (2S)-2Amino-3-(5' -(naphthalen-2" -yl)-1H-benzo[d][1.2.3] triazol-1' -yl)proponic acid (21e) was synthesized as described for (2S)-2-amino-3$\left[5^{\prime}\right.$-( $4^{\prime \prime}$-methoxyphenyl)-1H-benzo[d][1.2.3] triazol-1'-yl]propanoic acid hydrochloride (21a) using methyl (2S)-2-[(benzyloxycarbonyl)amino]-3-[ $5^{\prime}$-(naphthalen-2" -yl)- $1 H$-benzo [d] [1.2.3] triazol-1' -yl]propanoate $(19 \mathrm{e})(0.103 \mathrm{~g}, 0.213 \mathrm{mmol})$ and cesium carbonate $(0.0904 \mathrm{~g}, 0.277 \mathrm{mmol})$. Purification by recrystallization from methanol and diethyl ether gave (2S)-2-amino-3-[5'-(naphthalen$2^{\prime \prime}$-yl)-1H-benzo[d][1.2.3]triazol-1'-yl]propanoic acid hydrochloride (21e) as a white solid $(0.0994 \mathrm{~g}, 78 \%)$. mp $224-226{ }^{\circ} \mathrm{C}$; IR (neat) 2833, 1734, 1510, 1238,1143, $774 \mathrm{~cm}^{-1}$; $[\alpha]_{\mathrm{D}}^{20}+8.1$ (c 0.5, DMSO); ${ }^{1} \mathrm{H}$ NMR (400 MHz, $\left.\mathrm{CD}_{3} \mathrm{OD}\right): \delta 4.74(\mathrm{dd}, J=5.8,4.0 \mathrm{~Hz}, 1 \mathrm{H}), 5.33$ (dd, $J=15.4,4.0 \mathrm{~Hz}, 1 \mathrm{H}), 5.42(\mathrm{dd}, J=15.4,5.8 \mathrm{~Hz}, 1 \mathrm{H}), 7.38-7.66$ $(\mathrm{m}, 4 \mathrm{H}), 7.73(\mathrm{dd}, J=8.7,1.2 \mathrm{~Hz}, 1 \mathrm{H}), 7.76(\mathrm{~d}, J=8.5 \mathrm{~Hz}, 1 \mathrm{H})$, 7.89-8.05 (m, 3H), 8.09 (br s, $1 \mathrm{H}) ;{ }^{13} \mathrm{C}\left\{{ }^{1} \mathrm{H}\right\}$ NMR (101 MHz, $\left.\mathrm{CD}_{3} \mathrm{OD}\right): \delta 47.9\left(\mathrm{CH}_{2}\right), 52.7(\mathrm{CH}), 109.7(\mathrm{CH}), 119.5(\mathrm{CH}), 125.0$ $(\mathrm{CH}), 125.1(\mathrm{CH}), 125.7(\mathrm{CH}), 126.0(\mathrm{CH}), 127.1(\mathrm{CH}), 127.9$ (CH), $128.2(\mathrm{CH}), 130.7(\mathrm{CH}), 131.5(\mathrm{C}), 132.9(\mathrm{C}), 134.0(\mathrm{C})$, 137.9 (C), 138.9 (C), 145.8 (C), 168.1 (C); MS (ESI) $m / z: 355$ (M $\left.+\mathrm{Na}^{+}, 100\right)$; HRMS (ESI): calcd for $\mathrm{C}_{19} \mathrm{H}_{16} \mathrm{~N}_{4} \mathrm{NaO}_{2}\left(\mathrm{M}+\mathrm{Na}^{+}\right)$, 355.1165; found, 355.1155 .

(2S)-2-Amino-3-[5'-(thiophen-3"-yl)-1H-benzo[d][1.2.3]triazol-1'-yl]propanoic Acid Hydrochloride (21f). (2S)-2Amino-3-[5'-(thiophen-3"'-yl)-1H-benzo[d][1.2.3]triazol-1' -yl]propanoic acid hydrochloride (21f) was synthesized as described for (2S)-2-amino-3-[5'-(4"'-methoxyphenyl)-1H-benzo[d][1.2.3]triazol$1^{\prime}$-yl]propanoic acid hydrochloride (21a) using (2S)-2[(benzyloxycarbonyl)amino]-3-[5'-(thiophen-3"-yl)-1H-benzo[d][1.2.3] triazol-1'-yl]propanoate (19f) $(0.0810 \mathrm{~g}, 0.186 \mathrm{mmol})$ and cesium carbonate $(0.0785 \mathrm{~g}, 0.241 \mathrm{mmol})$. Purification by recrystallization from methanol and diethyl ether gave (2S)-2amino-3-[5' -(thiophen-3-yl)-1H-benzo[d][1.2.3] triazol-1' ${ }^{\prime}$-yl]propanoic acid hydrochloride (21f) as a white solid $(0.0453 \mathrm{~g}, 75 \%)$. $\mathrm{mp} 174-176{ }^{\circ} \mathrm{C}$; IR (neat) 2884, 1740, 1587, 1233, 1207, 775, 590 $\mathrm{cm}^{-1} ;[\alpha]_{\mathrm{D}}^{20}+12.5$ (c 0.5, DMSO); ${ }^{1} \mathrm{H}$ NMR (400 MHz, $\left.\mathrm{CD}_{3} \mathrm{OD}\right): \delta$ 4.81 (dd, $J=5.7,4.1 \mathrm{~Hz}, 1 \mathrm{H}), 5.27$ (dd, $J=15.5,4.1 \mathrm{~Hz}, 1 \mathrm{H}), 5.37$ $(\mathrm{dd}, J=15.5,5.7 \mathrm{~Hz}, 1 \mathrm{H}), 7.54(\mathrm{dd}, J=5.0,2.8 \mathrm{~Hz}, 1 \mathrm{H}), 7.56(\mathrm{dd}, J$ $=5.0,1.5 \mathrm{~Hz}, 1 \mathrm{H}), 7.76(\mathrm{dd}, J=2.8,1.5 \mathrm{~Hz}, 1 \mathrm{H}), 7.85(\mathrm{~d}, J=8.8 \mathrm{~Hz}$, $1 \mathrm{H}), 7.98(\mathrm{dd}, J=8.8,1.4 \mathrm{~Hz}, 1 \mathrm{H}), 8.26($ br s, $1 \mathrm{H}) ;{ }^{13} \mathrm{C}\left\{{ }^{1} \mathrm{H}\right\} \mathrm{NMR}$ $\left(101 \mathrm{MHz}, \mathrm{CD}_{3} \mathrm{OD}\right): \delta 46.7\left(\mathrm{CH}_{2}\right), 52.2(\mathrm{CH}), 110.1(\mathrm{CH}), 115.4$ $(\mathrm{CH}), 121.0(\mathrm{CH}), 125.9(\mathrm{CH}), 126.5(\mathrm{CH}), 127.6(\mathrm{CH}), 132.7$ (C), 133.3 (C), 141.0 (C), 146.2 (C), 167.5 (C); MS (ESI) m/z: 289 $\left(\mathrm{M}+\mathrm{H}^{+}, 100\right)$; HRMS (ESI): calcd for $\mathrm{C}_{13} \mathrm{H}_{13} \mathrm{~N}_{4} \mathrm{O}_{2} \mathrm{~S}\left(\mathrm{M}+\mathrm{H}^{+}\right)$, 289.0754; found, 289.0750 .

Methyl (2S)-2-[(Benzyloxycarbonyl)amino]-3-(7'-bromo-6'methoxy-1H-benzo[d][1.2.3]triazol-1'-yl)propanoate (22). To a dry flask under argon were added iron(III) chloride $(0.0030 \mathrm{~g}, 0.018$ $\mathrm{mmol})$ and $[\mathrm{BMIM}] \mathrm{NTf}_{2}(0.21 \mathrm{~mL}, 0.73 \mathrm{mmol})$, and the mixture was stirred for $0.5 \mathrm{~h}$. Methyl (2S)-2-[(benzyloxycarbonyl)amino]-3-(6'methoxy-1H-benzo[d][1.2.3] triazol-1'-yl)propanoate $(\mathbf{1 7 b})(0.14 \mathrm{~g}$, $0.36 \mathrm{mmol})$ was then added as a solution in dichloromethane (5.0 $\mathrm{mL})$, followed by NBS $(0.065 \mathrm{~g}, 0.36 \mathrm{mmol})$. After $1 \mathrm{~h}$ of stirring at room temperature, the mixture was diluted with ethyl acetate $(20 \mathrm{~mL})$ and filtered through a short pad of silica. The filtrate was washed with 
a $1 \mathrm{M}$ aqueous solution of sodium thiosulfate $(2 \times 20 \mathrm{~mL})$ and brine $(20 \mathrm{~mL})$, dried $\left(\mathrm{MgSO}_{4}\right)$, filtered, and concentrated in vacuo. Purification by flash column chromatography eluting with $10 \%$ ethyl acetate in dichloromethane gave methyl (2S)-2-[(benzyloxycarbonyl)amino]-3-(7'-bromo-6' -methoxy-1H-benzo[d][1.2.3] triazol-1'-yl)propanoate $(\mathbf{2 2})$ as a white solid $(0.14 \mathrm{~g}, 85 \%)$. mp $122-124{ }^{\circ} \mathrm{C}$; IR (neat) 3331, 2953, 1724, 1501, 1261, $1065 \mathrm{~cm}^{-1}$; $[\alpha]_{\mathrm{D}}^{23}-5.4(c 1.0$, $\left.\mathrm{CHCl}_{3}\right) ;{ }^{1} \mathrm{H}$ NMR $\left(500 \mathrm{MHz}, \mathrm{CDCl}_{3}\right): \delta 3.76(\mathrm{~s}, 3 \mathrm{H}), 4.00(\mathrm{~s}, 3 \mathrm{H})$, $4.96-5.12(\mathrm{~m}, 3 \mathrm{H}), 5.28(\mathrm{dd}, J=14.4,6.9 \mathrm{~Hz}, 1 \mathrm{H}), 5.47(\mathrm{dd}, J=$ $14.4,4.8 \mathrm{~Hz}, 1 \mathrm{H}), 5.68(\mathrm{~d}, J=8.1 \mathrm{~Hz}, 1 \mathrm{H}), 7.09(\mathrm{~d}, J=9.0 \mathrm{~Hz}, 1 \mathrm{H})$, 7.24-7.36 (m, 5H), $7.95(\mathrm{~d}, J=9.0 \mathrm{~Hz}, 1 \mathrm{H}) ;{ }^{13} \mathrm{C}\left\{{ }^{1} \mathrm{H}\right\}$ NMR $(126$ $\left.\mathrm{MHz}, \mathrm{CDCl}_{3}\right): \delta 49.8\left(\mathrm{CH}_{2}\right), 53.0\left(\mathrm{CH}_{3}\right), 54.4(\mathrm{CH}), 57.5\left(\mathrm{CH}_{3}\right)$, 67.2 $\left(\mathrm{CH}_{2}\right), 90.1(\mathrm{C}), 111.2(\mathrm{CH}), 119.8(\mathrm{CH}), 128.1(2 \times \mathrm{CH})$, $128.2(\mathrm{CH}), 128.5(2 \times \mathrm{CH}), 132.9(\mathrm{C}), 135.9(\mathrm{C}), 142.3(\mathrm{C}), 155.7$ (C), 156.2 (C), 169.7 (C); MS (ESI) $m / z: 485\left(\mathrm{M}+\mathrm{Na}^{+}, 100\right)$; HRMS (ESI): calcd for $\mathrm{C}_{19} \mathrm{H}_{19}{ }^{79} \mathrm{BrN}_{4} \mathrm{NaO}_{5}\left(\mathrm{M}+\mathrm{Na}^{+}\right)$, 485.0430; found, 485.0430 .

Methyl (2S)-2-[(Benzyloxycarbonyl)amino]-3-[6'-methoxy7'-(4"'-methoxyphenyl)-1H-benzo[d][1.2.3]triazol-1'-yl]propanoate (23). To a solution of methyl (2S)-2[(benzyloxycarbonyl)amino]-3-( $7^{\prime}$-bromo- $6^{\prime}$-methoxy- $1 H$-benzo $[d]$ [1.2.3] triazol-1'-yl)propanoate (22) $(0.140 \mathrm{~g}, 0.302 \mathrm{mmol})$ in $1,4-$ dioxane $(3.0 \mathrm{~mL})$ and water $(0.23 \mathrm{~mL})$ was added 4-methoxyphenylboronic acid $(0.0734 \mathrm{~g}, 0.483 \mathrm{mmol})$, potassium fluoride $(0.0526$ $\mathrm{g}, \quad 0.906 \mathrm{mmol})$, and [1,1'-bis(diphenylphosphino)ferrocene]dichloropalladium(II) (1:1) (0.0186 g, $0.0227 \mathrm{mmol})$. The mixture was degassed under argon for $0.25 \mathrm{~h}$ before heating to $80^{\circ} \mathrm{C}$ for $24 \mathrm{~h}$. The reaction was then cooled to ambient temperature and filtered through a pad of Celite, diluted with ethyl acetate $(20 \mathrm{~mL})$, and washed with water $(3 \times 20 \mathrm{~mL})$ and brine $(20 \mathrm{~mL})$. The organic layer was dried $\left(\mathrm{MgSO}_{4}\right)$, filtered, and concentrated in vacuo. Purification by flash column chromatography eluting with $10 \%$ ethyl acetate in dichloromethane gave methyl (2S)-2-[(benzyloxycarbonyl)amino]-3[6'-methoxy-7'-(4'"-methoxyphenyl)-1H-benzo[d][1.2.3] triazol-1' yl]propanoate $(23)$ as a pale orange oil $(0.0979 \mathrm{~g}, 66 \%)$. IR (neat) 3350, 2954, 1748, 1723,1519, 1497, 1288, 1065, 1028, $752 \mathrm{~cm}^{-1}$; $[\alpha]_{\mathrm{D}}^{20}-26.7\left(\mathrm{c} 1.0, \mathrm{CHCl}_{3}\right) ;{ }^{1} \mathrm{H}$ NMR $\left(400 \mathrm{MHz}, \mathrm{CDCl}_{3}\right): \delta 3.62(\mathrm{~s}$, $3 \mathrm{H}), 3.83(\mathrm{~s}, 3 \mathrm{H}), 3.88(\mathrm{~s}, 3 \mathrm{H}), 4.31(\mathrm{dd}, J=13.8,3.6 \mathrm{~Hz}, 1 \mathrm{H})$, $4.43-4.58(\mathrm{~m}, 2 \mathrm{H}), 4.98(\mathrm{~s}, 2 \mathrm{H}), 5.57(\mathrm{~d}, J=8.6 \mathrm{~Hz}, 1 \mathrm{H}), 6.97-7.07$ $(\mathrm{m}, 2 \mathrm{H}), 7.17(\mathrm{~d}, J=9.1 \mathrm{~Hz}, 1 \mathrm{H}), 7.21-7.38(\mathrm{~m}, 7 \mathrm{H}), 8.00(\mathrm{~d}, J=$ $9.1 \mathrm{~Hz}, 1 \mathrm{H}) ;{ }^{13} \mathrm{C}\left\{{ }^{1} \mathrm{H}\right\}$ NMR $\left(101 \mathrm{MHz}, \mathrm{CDCl}_{3}\right): \delta 49.9\left(\mathrm{CH}_{2}\right), 52.7$ $\left(\mathrm{CH}_{3}\right), 53.6(\mathrm{CH}), 55.3\left(\mathrm{CH}_{3}\right), 57.2\left(\mathrm{CH}_{3}\right), 67.1\left(\mathrm{CH}_{2}\right), 111.2$ $(\mathrm{CH}), 111.8(\mathrm{C}), 114.0(\mathrm{CH}), 114.2(\mathrm{CH}), 119.7(\mathrm{CH}), 124.5(\mathrm{C})$, $127.9(2 \times \mathrm{CH}), 128.1(\mathrm{CH}), 128.5(2 \times \mathrm{CH}), 131.7(\mathrm{CH}), 131.8$ (CH), $133.0(\mathrm{C}), 136.0$ (C), 141.7 (C), 155.6 (C), 156.7 (C), 159.6 (C), 169.6 (C); MS (EI) m/z: $490\left(\mathrm{M}^{+}, 24\right), 240$ (94), 227 (42), 212 (37), 108 (52), 91 (100), 79 (49); HRMS (EI) calcd for $\mathrm{C}_{26} \mathrm{H}_{26} \mathrm{~N}_{4} \mathrm{O}_{6}$ $\left(\mathrm{M}^{+}\right)$, 490.1852; found, 490.1831.

(2S)-2-Amino-3-(6'-methoxy-7'-(4" -methoxyphenyl)-1 Hbenzo[d][1.2.3]triazol-1'-yl)propanoic Acid Hydrochloride (24). To a solution of methyl (2S)-2-[(benzyloxycarbonyl)amino]3-[6'-methoxy-7' -(4" -methoxyphenyl)-1H-benzo[ d] [1.2.3] triazol-1' yl]propanoate $(23)(0.097 \mathrm{~g}, 0.198 \mathrm{mmol})$ in methanol $(7.0 \mathrm{~mL})$ and water $(3.0 \mathrm{~mL})$ was added cesium carbonate $(0.084 \mathrm{~g}, 0.257 \mathrm{mmol})$, and the reaction mixture was stirred at room temperature for $16 \mathrm{~h}$. The reaction mixture was concentrated in vacuo and dissolved in water $(5.0 \mathrm{~mL})$ before the addition of $1 \mathrm{M}$ aqueous hydrochloric acid solution $(10 \mathrm{~mL})$. The product was extracted with dichloromethane $(3 \times 20 \mathrm{~mL})$, dried $\left(\mathrm{MgSO}_{4}\right)$, filtered, and concentrated in vacuo to afford (2S)-2-[(benzyloxycarbonyl)amino]-3-[6'-methoxy-7'-(4"'-methoxyphenyl)-1H-benzo[ $d][1.2 .3]$ triazol- $1^{\prime}$-yl $]$ propanoic acid as a yellow foam $(0.089 \mathrm{~g}, 94 \%)$. This material was then used immediately in the following step. To a solution of (2S)-2-[(benzyloxycarbonyl)amino]-3-[6'-methoxy-7'-(4"'-methoxyphenyl)-1H-benzo[d][1.2.3]triazol-1'-yl]propanoic acid $(0.044 \mathrm{~g}, 0.092 \mathrm{mmol})$ in tetrahydrofuran $(5 \mathrm{~mL})$ was added $10 \%$ palladium on carbon $(0.019 \mathrm{~g})$, and the reaction mixture was purged under hydrogen gas for $1 \mathrm{~h}$, before stirring at room temperature under a hydrogen atmosphere for $16 \mathrm{~h}$. The reaction mixture was added to a $1 \mathrm{M}$ aqueous solution of hydrochloric acid $(0.50 \mathrm{~mL})$, filtered through Celite, and washed with methanol $(10 \mathrm{~mL})$, and the filtrate was concentrated in vacuo. Purification by recrystallization from methanol and diethyl ether gave (2S)-2-amino-3-(6'-methoxy-7' - (4" -methoxyphenyl)-1H-benzo[d]-

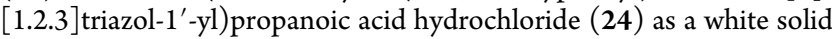
(0.023 g, 73\%). mp 130-132 ${ }^{\circ} \mathrm{C}$; IR (neat) 3698, 2931, 1686, 1595, $1354,1252,1169,984 \mathrm{~cm}^{-1} ;[\alpha]_{\mathrm{D}}^{19}-6.2(c 0.1, \mathrm{MeOH}) ;{ }^{1} \mathrm{H}$ NMR $\left(400 \mathrm{MHz}, \mathrm{CD}_{3} \mathrm{OD}\right): \delta 3.83(\mathrm{~s}, 3 \mathrm{H}), 3.85-3.97(\mathrm{~m}, 4 \mathrm{H}), 4.43$ (dd, $J$ $=15.2,9.6 \mathrm{~Hz}, 1 \mathrm{H}), 4.57(\mathrm{dd}, J=15.2,2.5 \mathrm{~Hz}, 1 \mathrm{H}), 7.00-7.12(\mathrm{~m}$, $2 \mathrm{H}), 7.23-7.34(\mathrm{~m}, 2 \mathrm{H}), 7.37(\mathrm{~d}, J=9.1 \mathrm{~Hz}, 1 \mathrm{H}), 7.99(\mathrm{~d}, J=9.1$ $\mathrm{Hz}, 1 \mathrm{H}) ;{ }^{13} \mathrm{C}\left\{{ }^{1} \mathrm{H}\right\}$ NMR $\left(126 \mathrm{MHz}, \mathrm{CD}_{3} \mathrm{OD}\right): \delta 50.8\left(\mathrm{CH}_{2}\right), 55.1$ $(\mathrm{CH}), 55.8\left(\mathrm{CH}_{3}\right), 57.7\left(\mathrm{CH}_{3}\right), 113.5(\mathrm{CH}), 113.9(\mathrm{C}), 115.2(\mathrm{CH})$, $115.3(\mathrm{CH}), 120.0(\mathrm{CH}), 125.6(\mathrm{C}), 132.8(\mathrm{CH}), 133.2(\mathrm{CH}), 134.3$ (C), 142.7 (C), 158.6 (C), 161.3 (C), 170.2 (C); MS (ESI) $m / z: 365$ $\left(\mathrm{M}+\mathrm{Na}^{+}, 100\right)$; HRMS (ESI): calcd for $\mathrm{C}_{17} \mathrm{H}_{18} \mathrm{~N}_{4} \mathrm{NaO}_{4}\left(\mathrm{M}+\mathrm{Na}^{+}\right)$, 365.1220; found, 365.1210 .

\section{ASSOCIATED CONTENT}

\section{S Supporting Information}

The Supporting Information is available free of charge on the ACS Publications website at DOI: 10.1021/acs.joc.9b01685.

Absorption and emission spectra for $\alpha$-amino acids 21a-c, 21e, 21f, and 24 and ${ }^{1} \mathrm{H}$ and ${ }^{13} \mathrm{C}$ NMR spectra for all novel compounds (PDF)

\section{AUTHOR INFORMATION}

\section{Corresponding Author}

*E-mail: Andrew.Sutherland@glasgow.ac.uk.

ORCID

Andrew Sutherland: 0000-0001-7907-5766

Notes

The authors declare no competing financial interest.

\section{ACKNOWLEDGMENTS}

Financial support from EPSRC (studentships to J.D.B., EP/ N509176/1, T.E.F.M., EP/M506539/1 and A.H.H., EP/ K503058/1), GlaxoSmithKline Ltd, the Neurosciences Foundation and the University of Glasgow is gratefully acknowledged.

\section{REFERENCES}

(1) For reviews see: (a) Wagner, I.; Musso, H. New Naturally Occurring Amino Acids. Angew. Chem., Int. Ed. Engl. 1983, 22, 816828. (b) Greenstein, J. P.; Winitz, M. Chemistry of the Amino Acids; Krieger, R. E.: FL, 1984; Vol. 1-3. (c) Chemistry and Biochemistry of the Amino Acids; Barrett, G. E., Ed.; Chapman and Hall: London, 1985. (d) Breslow, R.; Chmielewski, J.; Foley, D.; Johnson, B.; Kumabe, N.; Varney, M.; Mehra, R. Optically Active Amino Acid Synthesis by Artificial Transaminase Enzymes. Tetrahedron 1988, 44, 5515-5524.

(2) (a) Ohfune, Y. Stereoselective Routes Towards the Synthesis of Unusual Amino Acids. Acc. Chem. Res. 1992, 25, 360-366. (b) Easton, C. J. Free-Radical Reactions in the Synthesis of $\alpha$-Amino Acids and Derivatives. Chem. Rev. 1997, 97, 53-82. (c) Vogt, H.; Bräse, S. Recent Approaches Towards the Asymmetric Synthesis of $\alpha, \alpha$ Disubstituted $\alpha$-Amino Acids. Org. Biomol. Chem. 2007, 5, 406-430. (d) Nájera, C.; Sansano, J. M. Catalytic Asymmetric Synthesis of $\alpha$ Amino Acids. Chem. Rev. 2007, 107, 4584-4671. (e) Smits, R.; Cadicamo, C. D.; Burger, K.; Koksch, B. Synthetic Strategies to $\alpha$ Trifluoromethyl and $\alpha$-Difluoromethyl Substituted $\alpha$-Amino Acids. Chem. Soc. Rev. 2008, 37, 1727-1739. (f) Mortensen, M.; Husmann, R.; Veri, E.; Bolm, C. Synthesis and Applications of SiliconContaining $\alpha$-Amino Acids. Chem. Soc. Rev. 2009, 38, 1002-1010. (g) Noisier, A. F. M.; Brimble, M. A. C-H Functionalization in the Synthesis of Amino Acids and Peptides. Chem. Rev. 2014, 114, 87758806. 
(3) (a) Dougherty, D. Unnatural Amino Acids as Probes of Protein Structure and Function. Curr. Opin. Chem. Biol. 2000, 4, 645-652. (b) Walsh, C. T.; O’Brien, R. V.; Khosla, C. Nonproteinogenic Amino Acid Building Blocks for Nonribosomal Peptide and Hybrid Polyketide Scaffolds. Angew. Chem., Int. Ed. 2013, 52, 7098-7124. (c) Lang, K.; Chin, J. W. Cellular Incorporation of Unnatural Amino Acids and Bioorthogonal Labeling of Proteins. Chem. Rev. 2014, 114, 4764-4806.

(4) For recent reviews of fluorescent amino acids, see: (a) Sinkeldam, R. W.; Greco, N. J.; Tor, Y. Fluorescent Analogs of Biomolecular Building Blocks: Design, Properties and Applications. Chem. Rev. 2010, 110, 2579-2619. (b) Krueger, A. T.; Imperiali, B. Fluorescent Amino Acids: Modular Building Blocks for the Assembly of New Tools for Chemical Biology. ChemBioChem 2013, 14, 788-799. (c) Kubota, R.; Hamachi, I. Protein Recognition using Synthetic Small-Molecular Binders Toward Optical Protein Sensing In Vitro and in Live Cells. Chem. Soc. Rev. 2015, 44, 4454-4471. (d) Harkiss, A. H.; Sutherland, A. Recent Advances in the Synthesis and Application of Fluorescent $\alpha$-Amino Acids. Org. Biomol. Chem. 2016, 14, 89118921.

(5) (a) Benedetti, E.; Veliz, A. B. E.; Charpenay, M.; Kocsis, L. S.; Brummond, K. M. Attachable Solvatochromic Fluorophores and Bioconjugation Studies. Org. Lett. 2013, 15, 2578-2581. (b) Ruan, Y.-B.; Yu, Y.; Li, C.; Bogliotti, N.; Tang, J.; Xie, J. Triazolyl Benzothiadiazole Fluorescent Chemosensors: A Systematic Investigation of 1,4- or 1,5-Disubstituted Mono- and Bis-triazole Derivatives. Tetrahedron 2013, 69, 4603-4608. (c) Häußler, D.; Gütschow, M. Fluorescently Labeled Amino Acids as Building Blocks for Bioactive Molecules. Synthesis 2016, 48, 245-255.

(6) Vázquez, M. E.; Rothman, D. M.; Imperiali, B. A New Environment-Sensitive Fluorescent Amino Acid for Fmoc-Based Solid Phase Peptide Synthesis. Org. Biomol. Chem. 2004, 2, 1965-1966.

(7) Loving, G.; Imperiali, B. A Versatile Amino Acid Analogue of the Solvatochromic Fluorophore 4-N,N-Dimethylamino-1,8-Naphthalimide: A Powerful Tool for the Study of Dynamic Protein Interactions. J. Am. Chem. Soc. 2008, 130, 13630-13638.

(8) (a) Lee, H. S.; Guo, J.; Lemke, E. A.; Dimla, R. D.; Schultz, P. G. Genetic Incorporation of a Small, Environmentally Sensitive, Fluorescent Probe into Proteins in Saccharomyces cerevisiae. J. Am. Chem. Soc. 2009, 131, 12921-12923. (b) Xiang, Z.; Wang, L. Enantiospecific Synthesis of Genetically Encodable Fluorescent Unnatural Amino Acid L-3-(6-Acetylnaphthalen-2-ylamino)-2-aminopropanoic Acid. J. Org. Chem. 2011, 76, 6367-6371. (c) Chatterjee, A.; Guo, J.; Lee, H. S.; Schultz, P. G. A Genetically Encoded Fluorescent Probe in Mammalian Cells. J. Am. Chem. Soc. 2013, 135, 12540-12543. (d) Wen, M.; Guo, X.; Sun, P.; Xiao, L.; Li, J.; Xiong, Y.; Bao, J.; Xue, T.; Zhang, L.; Tian, C. Site-Specific Fluorescence Spectrum Detection and Characterization of hASICa Channels Upon Toxin Mambalgin-1 Binding in Live Mammalian Cells. Chem. Commun. 2015, 51, 8153-8156.

(9) (a) Turcatti, G.; Zoffmann, S.; Lowe, J. A., III; Drozda, S. E.; Chassaing, G.; Schwartz, T. W.; Chollet, A. Characterization of Nonpeptide Antagonist and Peptide Agonist Binding Sites of the NK1 Receptor with Fluorescent Ligands. J. Biol. Chem. 1997, 272, 2116721175. (b) Summerer, D.; Chen, S.; Wu, N.; Deiters, A.; Chin, J. W. A Genetically Encoded Fluorescent Amino Acid. PNAS 2006, 103, 9785-9789.

(10) (a) Schultz, G.; Nemeth, K.; Edgerton, M. D.; Meseth, U.; Talabot, F.; Peitsch, M.; Knowles, J.; Vogel, H.; Chollet, A. Probing the Structure and Function of the Tachykinin Neurokinin-2 Receptor Through Biosynthetic Incorporation of Fluorescent Amino Acids at Specific Sites. J. Biol. Chem. 1996, 271, 19991-19998. (b) Dufau, I.; Mazarguil, H. Design of a Fluorescent Amino Acid Derivative Useable in Peptide Synthesis. Tetrahedron Lett. 2000, 41, 6063-6066.

(11) Li, C.; Henry, E.; Mani, N. K.; Tang, J.; Brochon, J.-C.; Deprez, E. Click Chemistry to Fluorescent Amino Esters: Synthesis and Spectroscopic Studies. Eur. J. Org. Chem. 2010, 2010, 2395-2405.

(12) (a) Fowler, L. S.; Ellis, D.; Sutherland, A. Synthesis of Fluorescent Enone Derived $\alpha$-Amino Acids. Org. Biomol. Chem. 2009,
7, 4309-4316. (b) Gilfillan, L.; Artschwager, R.; Harkiss, A. H.; Liskamp, R. M. J.; Sutherland, A. Synthesis of Pyrazole Containing $\alpha$ Amino Acids via a Highly Regioselective Condensation/aza-Michael Reaction of $\beta$-Aryl $\alpha, \beta$-Unsaturated Ketones. Org. Biomol. Chem. 2015, 13, 4514-4523. (c) Harkiss, A. H.; Bell, J. D.; Knuhtsen, A.; Jamieson, A. G.; Sutherland, A. Synthesis and Fluorescent Properties of $\beta$-Pyridyl $\alpha$-Amino Acids. J. Org. Chem. 2019, 84, 2879-2890.

(13) (a) Filimonov, V. D.; Trusova, M.; Postnikov, P.; Krasnokutskaya, E. A.; Lee, Y. M.; Hwang, H. Y.; Kim, H.; Chi, K.W. Unusually Stable, Versatile and Pure Arenediazonium Tosylates: Their Preparation, Structures and Synthetic Applicability. Org. Lett. 2008, 10, 3961-3964. (b) Kutonova, K. V.; Trusova, M. E.; Stankevich, A. V.; Postnikov, P. S.; Filimonov, V. D. Matsuda-Heck Reaction with Arenediazonium Tosylates in Water. Beilstein J. Org. Chem. 2015, 11, 358-362. (c) Kutonova, K. V.; Jung, N.; Trusova, M. E.; Filimonov, V. D.; Postnikov, P. S.; Bräse, S. Arenediazonium Tosylates (ADTs) as Efficient Reagents for Suzuki-Miyaura CrossCoupling in Neat Water. Synthesis 2017, 49, 1680-1688.

(14) (a) Sloan, N. L.; Luthra, S. K.; McRobbie, G.; Pimlott, S. L.; Sutherland, A. A One-Pot Radioiodination of Aryl Amines via Stable Diazonium Salts: Preparation of ${ }^{125}$ I-Imaging Agents. Chem. Commun. 2017, 53, 11008-11011. (b) Trusova, M. E.; Rodriguez-Zubiri, M.; Kutonova, K. V.; Jung, N.; Bräse, S.; Felpin, F.-X.; Postnikov, P. S. Ultra-Fast Suzuki and Heck Reactions for the Synthesis of Styrenes and Stilbenes using Arenediazonium Salts as Super-Electrophiles. Org. Chem. Front. 2018, 5, 41-45. (c) Faggyas, R. J.; Grace, M.; Williams, L.; Sutherland, A. Multibond Forming Tandem Reactions of Anilines via Stable Aryl Diazonium Salts: One-Pot Synthesis of 3,4Dihydroquinolin-2-ones. J. Org. Chem. 2018, 83, 12595-12608.

(15) Faggyas, R. J.; Sloan, N. L.; Buijs, N.; Sutherland, A. Synthesis of Structurally Diverse Benzotriazoles via Rapid Diazotization and Intramolecular Cyclization of 1,2-Aryldiamines. Eur. J. Org. Chem. 2019; 4 DOI: 10.1002/ejoc.201900463.

(16) Zhang, L.-h.; Kauffman, G. S.; Pesti, J. A.; Yin, J. Rearrangement of $\mathrm{N}_{\alpha}$-Protected L-Asparagines with Iodosobenzene Diacetate. A Practical Route to $\beta$-Amino-L-alanine Derivatives. J. Org. Chem. 1997, 62, 6918-6920.

(17) Egbertson, M. S.; Cook, J. J.; Bednar, B.; Prugh, J. D.; Bednar, R. A.; Gaul, S. L.; Gould, R. J.; Hartman, G. D.; Homnick, C. F.; Holahan, M. A.; Libby, L. A.; Lynch, J. J., Jr.; Lynch, R. J.; Stranieri, M. T.; Vassallo, L. M. Non-Peptide GPIIb/IIIa Inhibitors. 20. Centrally Constrained Thienothiophene $\alpha$-Sulfonamides Are Potent, Long Acting in Vivo Inhibitors of Platelet Aggregation. J. Med. Chem. 1999, 42, 2409-2421.

(18) Huber, V. J.; Arroll, T. W.; Lum, C.; Goodman, B. A.; Nakanishi, H. (1-Benzimidazolonyl)alanine (Bia): Preliminary Investigations into a Potential Tryptophan Mimetic. Tetrahedron Lett. 2002, 43, 6729-6733.

(19) Further attempts at optimizing this transformation investigated lower reaction temperatures. However, this led to incomplete conversions and lower overall yields of $\mathbf{1 7} \mathbf{b}$.

(20) Miyaura, N.; Suzuki, A. Palladium-Catalyzed Cross-Coupling Reactions of Organoboron Compounds. Chem. Rev. 1995, 95, 24572483.

(21) (a) Racys, D. T.; Warrilow, C. E.; Pimlott, S. L.; Sutherland, A. Highly Regioselective Iodination of Arenes via Iron(III)-Catalyzed Activation of N-Iodosuccinimide. Org. Lett. 2015, 17, 4782-4785. (b) Mostafa, M. A. B.; Calder, E. D. D.; Racys, D. T.; Sutherland, A. Intermolecular Aryl C-H Amination Through Sequential Iron and Copper Catalysis. Chem.-Eur. J. 2017, 23, 1044-1047. (c) Mostafa, M. A. B.; Bowley, R. M.; Racys, D. T.; Henry, M. C.; Sutherland, A. Iron(III)-Catalyzed Chlorination of Activated Arenes. J. Org. Chem. 2017, 82, 7529-7537.

(22) Henry, M. C.; Senn, H. M.; Sutherland, A. Synthesis of Functionalized Indolines and Dihydrobenzofurans by Iron and Copper Catalyzed Aryl C-N and C-O Bond Formation. J. Org. Chem. 2019, 84, 346-364.

(23) The absorption and fluorescence spectra for $\alpha$-amino acids $21 \mathrm{a}-\mathrm{c}, \mathbf{2 1 e}, \mathbf{2 1 f}$ and 24 are presented in the Supporting Information. 
(24) (a) Bordeau, G.; Lartia, R.; Metge, G.; Fiorini-Debuisschert, C.; Charra, F.; Teulade-Fichou, M.-P. Trinaphthylamines as Robust Organic Materials for Two-Photon-Induced Fluorescence. J. Am. Chem. Soc. 2008, 130, 16836-16837. (b) Achelle, S.; Nouira, I.; Pfaffinger, B.; Ramondenc, Y.; Plé, N.; Rodríguez-López, J. V-Shaped 4,6-Bis(arylvinyl)pyrimidine Oligomers: Synthesis and Optical Properties. J. Org. Chem. 2009, 74, 3711-3717.

(25) Galievsky, V. A.; Druzhinin, S. I.; Demeter, A.; Kovalenko, S. A.; Senyushkina, T.; Mayer, P.; Zachariasse, K. A. Presence and Absence of Excited State Intramolecular Charge Transfer with the Six Isomers of Dicyano- $N, N$-dimethylaniline and Dicyano-( $N$-methyl- $N$ isopropyl)aniline. J. Phys. Chem. A 2011, 115, 10823-10845. 